\title{
$\mu$-VALUES AND SPECTRAL VALUE SETS FOR LINEAR PERTURBATION CLASSES DEFINED BY A SCALAR PRODUCT*
}

\author{
MICHAEL KAROW
}

\begin{abstract}
We study the variation of the spectrum of matrices under perturbations which are self- or skew-adjoint with respect to a scalar product. Computable formulas are given for the associated $\mu$-values. The results can be used to calculate spectral value sets for the perturbation classes under consideration. We discuss the special case of complex Hamiltonian perturbations of a Hamiltonian matrix in detail.
\end{abstract}

Key words. linear systems, eigenvalues, perturbations, spectral value sets, $\boldsymbol{\mu}$-values

AMS subject classifications. 15A18, 15A57, 15A63, 93C05, 93C73

DOI. $10.1137 / 090774896$

1. Introduction. $\mu$-values are well established tools in stability analysis of uncertain systems and in eigenvalue perturbation theory [5], [8], [10], [20], [25]. They can be used to characterize several important quantities including stability radii and structured eigenvalue condition numbers [11],[15]. The relationship of spectral value sets (also known as structured pseudospectra) with $\mu$-values will be shown below. There is a vast literature on the problem of calculating $\mu$-values with respect to various perturbation classes [1], [2], [4], [6], [12], [14], [22], [23], [24]. In this paper we give computable formulas for $\mu$ if the underlying perturbation class is a set of self-adjoint or skew-adjoint matrices with respect to a scalar product. The scalar product is assumed to be defined by a unitary matrix; see section 4 . It will be shown that in this case the associated $\mu$-values can be obtained by minimizing a univariate and unimodular function. The formulas presented in this paper have been applied in the software package Structured EigToo ${ }^{1}$ in order to compute structured pseudospectra with respect to skew-symmetric, Hermitian, and Hamiltonian perturbations; see [13].

We use the following notation. The symbols $\mathbb{R}$ and $\mathbb{C}$ represent the sets of real and complex numbers, respectively. By $\mathbb{C}^{n \times m}$ we denote the set of $n$ by $m$ matrices with entries in $\mathbb{C}$. Furthermore, $\mathbb{C}^{n}=\mathbb{C}^{n \times 1}$ is the set of column vectors of length $n$. The conjugate, the transpose, and the conjugate transpose of $A \in \mathbb{C}^{n \times m}$ will be written $\bar{A}, A^{\top}$, and $A^{*}$. If $A$ is square, then $\sigma(A)$ and $\rho(A)=\mathbb{C} \backslash \sigma(A)$ denote its spectrum and its resolvent set. The identity matrix of size $n$ will be denoted by $I_{n}$. We drop the index $n$ if the size is clear from the context. The real and the imaginary part of $z \in \mathbb{C}$ are written as $\mathfrak{R} z$ and $\mathfrak{\Im} z$, respectively.

By a perturbation class $\Delta$ we mean a nonempty closed subset of $\mathbb{C}^{l \times q}$ which is star shaped with respect to $0 \in \mathbb{C}^{l \times q}$; i.e., if $\Delta \in \boldsymbol{\Delta}$, then $t \Delta \in \boldsymbol{\Delta}$ for $0 \leq t \leq 1$. We now give the definition of $\mu$-values.

Definition 1.1. Let $\boldsymbol{\Delta} \subseteq \mathbb{C}^{l \times q}$ be a perturbation class, and let $\|\cdot\|$ be a norm on $\mathbb{C}^{l \times q}$.

(i) The $\mu$-value of $M \in \mathbb{C}^{q \times l}$ with respect to $\Delta$ and $\|\cdot\|$ is

$$
\mu_{\Delta}(M):=(\inf \{\|\Delta\| ; \Delta \in \Delta, 1 \in \sigma(\Delta M)\})^{-1} .
$$

*Received by the editors October 26, 2009; accepted for publication (in revised form) by N. J. Higham May 25, 2011; published electronically September 6, 2011.

http://www.siam.org/journals/simax/32-3/77489.html

'Mathematics Institute, Berlin University of Technology, D-10623 Berlin, Germany, (karow@math. TU-Berlin.de).

${ }^{1}$ Available from http://www.sam.math.ethz.ch/NLAgroup/software.html. 
Thus, $\mu_{\Delta}(M)$ is the inverse of the smallest norm of a $\Delta \in \Delta$ such that 1 is an eigenvalue of the matrix product $\Delta M$. If there is no such $\Delta \in \Delta$, then $\mu_{\Delta}(M)=0$.

(ii) If $l=q$, then the $\mu$-value of $M$ of second kind is defined as

$$
\tilde{\mu}_{\Delta}(M):=\inf \{\|\Delta\| ; \Delta \in \Delta, \operatorname{det}(M-\Delta)=0\} .
$$

Thus, $\tilde{\mu}_{\Delta}(M)$ is the structured distance of $M$ to the set of singular matrices. We have $\tilde{\mu}_{\Delta}(M)=0$ iff $M$ is singular and $\tilde{\mu}_{\Delta}(M)=\infty$ iff there is no $\Delta \in \Delta$ such that $\operatorname{det}(M-\Delta)=0$.

It is easy to see that $\tilde{\mu}_{\Delta}(M)=\mu_{\Delta}\left(M^{-1}\right)^{-1}$ if $M$ is nonsingular. Furthermore, if the underlying norm is the spectral norm, then

$$
\begin{aligned}
\mu_{\mathbb{C}^{l \times q}}(M)=\sigma_{\max }(M) & \text { for all } M \in \mathbb{C}^{l \times q}, \\
\tilde{\mu}_{\mathbb{C}^{n \times n}}(M)=\sigma_{\min }(M) & \text { for all } M \in \mathbb{C}^{n \times n},
\end{aligned}
$$

where $\sigma_{\max }(\cdot)$ and $\sigma_{\min }(\cdot)$ denote the maximum and the minimum singular value, respectively.

We now briefly discuss the relationship of $\mu$-values with the perturbation analysis of eigenvalues. Consider matrix perturbations of the form

$$
A \rightsquigarrow A_{\Delta}=A+B \Delta C, \quad \Delta \in \Delta, \quad\|\Delta\|<\delta,
$$

where $A \in \mathbb{C}^{n \times n}, B \in \mathbb{C}^{n \times l}, C \in \mathbb{C}^{q \times n}$ are fixed matrices. The set of all eigenvalues of all matrices $A_{\Delta}$ given by (1.4) is called a spectral value set (stuctured pseudospectrum). It is denoted by

$$
\begin{aligned}
\sigma_{\Delta}(A, B, C, \delta): & =\bigcup_{\Delta \in \Delta,\|\Delta\|<\delta} \sigma(A+B \Delta C) \\
& =\{s \in \mathbb{C} ; \exists \Delta \in \Delta:\|\Delta\|<\delta, \text { and } \operatorname{det}(s I-(A+B \Delta C))=0\} .
\end{aligned}
$$

Let $G(s):=C(s I-A)^{-1} B, s \in \rho(A)$, be the transfer function of the triple $(A, B, C)$. From the well known equivalence [7, Proposition 2.3]

$$
s \in \sigma(A+B \Delta C) \Leftrightarrow 1 \in \sigma(\Delta G(s))
$$

it follows that

$$
\mu_{\Delta}(G(s))=(\inf \{\|\Delta\| ; \Delta \in \Delta, s \in \sigma(A+B \Delta C)\})^{-1}, \quad s \in \rho(A) .
$$

This in turn yields

$$
\sigma_{\Delta}(A, B, C, \delta)=\sigma(A) \cup\left\{s \in \rho(A) ; \mu_{\Delta}(G(s))>\delta^{-1}\right\}, \quad \delta>0 .
$$

For the cases $B=C=I$ and $\Delta \subseteq \mathbb{C}^{n \times n}$ we simplify notation and denote the associated spectral value sets by

$$
\sigma_{\Delta}(A, \delta):=\sigma_{\Delta}(A, I, I, \delta)=\bigcup_{\Delta \in \Delta,\|\Delta\|<\delta} \sigma(A+\Delta) .
$$

From the definition of $\tilde{\mu}$ it is immediate that, for $A \in \mathbb{C}^{n \times n}$,

Copyright ( by SIAM. Unauthorized reproduction of this article is prohibited. 


$$
\begin{aligned}
\tilde{\mu}_{\Delta}(s I-A) & =\inf \{\|\Delta\| \mid \Delta \in \Delta, s \in \sigma(A+\Delta)\}, \quad s \in \mathbb{C}, \\
\sigma_{\Delta}(A, \delta) & =\left\{s \in \mathbb{C} ; \tilde{\mu}_{\Delta}(s I-A)<\delta\right\}, \quad \delta>0 .
\end{aligned}
$$

The statements (1.8) and (1.11) yield that spectral value sets can be calculated by evaluating the functions $s \mapsto \mu_{\Delta}(G(s))$ and $s \mapsto \tilde{\mu}_{\Delta}(s I-A)$, respectively.

The organization of this paper is as follows. In section 2 we provide useful characterizations for $\mu$ with respect to Hermitian, complex symmetric, and complex skewsymmetric perturbations. In particular we show that the $\mu$-value and the $\tilde{\mu}$-value for symmetric perturbations coincide with the $\mu$-value and the $\tilde{\mu}$-value for unstructured perturbations, i.e., with the maximum and the minimum singular value. Therefore, symmetric perturbations are not considered in the following sections. Section 3 contains the main results of this paper. Here, we show how $\mu$-values with respect to Hermitian and skew-symmetric perturbations can be computed by maximizing or minimizing a certain eigenvalue of a Hermitian pencil. The technical proofs of the main results are given in section 6 . In section 4 we treat $\mu$-values for perturbation classes of self- and skew-adjoint matrices with respect to a scalar product. Section 5 deals with a special case: $\mu$-values and spectral value sets for Hamiltonian perturbations of Hamiltonian matrices.

Throughout the rest of this paper the underlying norm $\|\cdot\|$ is the spectral norm.

2. Hermitian, symmetric, and skew-symmetric perturbations. In this section we derive basic characterizations for $\mu$-values with respect to the perturbation classes

$$
\Delta \in\{\operatorname{Herm}(n), \operatorname{Sym}(n), \operatorname{Skew}(n)\},
$$

where

$$
\begin{aligned}
\operatorname{Herm}(n) & :=\left\{\Delta \in \mathbb{C}^{n \times n} ; \Delta^{*}=\Delta\right\}, \\
\operatorname{Sym}(n) & :=\left\{\Delta \in \mathbb{C}^{n \times n} ; \Delta^{\top}=\Delta\right\}, \\
\operatorname{Skew}(n) & :=\left\{\Delta \in \mathbb{C}^{n \times n} ; \Delta^{\top}=-\Delta\right\} .
\end{aligned}
$$

Theorem 2.1. Let $M \in \mathbb{C}^{n \times n}$. Then the following statements hold.

(a) If the Hermitian matrix $M_{h}=i\left(M-M^{*}\right)$ is positive or negative definite, then $\operatorname{det}(M-\Delta) \neq 0$ and $\operatorname{det}(\Delta M-I) \neq 0$ for all $\Delta \in \operatorname{Herm}(n)$. Hence, $\tilde{\mu}_{\mathrm{Herm}}(M)=\infty$ and $\mu_{\mathrm{Herm}}(M)=0$. If $M_{h}$ is not definite, then

$$
\begin{aligned}
& \mu_{\text {Herm }}(M)=\max \left\{\|M v\| ; v \in \mathbb{C}^{n},\|v\|=1, v^{*} M_{h} v=0\right\}, \\
& \tilde{\mu}_{\text {Herm }}(M)=\min \left\{\|M v\| ; v \in \mathbb{C}^{n},\|v\|=1, v^{*} M_{h} v=0\right\} .
\end{aligned}
$$

(b) Let $M_{s}=M+M^{\top}$. Then, for $n \geq 2$,

$$
\begin{aligned}
& \mu_{\text {Skew }}(M)=\max \left\{\|M v\| ; v \in \mathbb{C}^{n},\|v\|=1, v^{\top} M_{s} v=0\right\}, \\
& \tilde{\mu}_{\text {Skew }}(M)=\min \left\{\|M v\| ; v \in \mathbb{C}^{n},\|v\|=1, v^{\top} M_{s} v=0\right\} .
\end{aligned}
$$

(c) We always have

$$
\begin{aligned}
& \mu_{\text {Sym }}(M)=\max \left\{\|M v\| ; v \in \mathbb{C}^{n},\|v\|=1\right\}=\sigma_{\max }(M), \\
& \tilde{\mu}_{\text {Sym }}(M)=\min \left\{\|M v\| ; v \in \mathbb{C}^{n},\|v\|=1\right\}=\sigma_{\min }(M),
\end{aligned}
$$

Copyright $@$ by SIAM. Unauthorized reproduction of this article is prohibited. 
where $\sigma_{\max }(\cdot)$ and $\sigma_{\min }(\cdot)$ denote the maximum and the minimum singular values.

Note that the $\mu$-values for the symmetric case coincide with the $\mu$-values for the unstructured case $\Delta=\mathbb{C}^{n \times n}$ (see relation (1.3)).

Proof. For $\Delta \subseteq \mathbb{C}^{n \times n}$ and $x, y \in \mathbb{C}^{n}$ let $v_{\Delta}(x, y):=\inf \{\|\Delta\| ; \Delta \in \Delta, \Delta x=y\}$. The relation $\Delta x=y$ implies $\|\Delta\|\|x\| \geq\|y\|$. Thus, for all $x \neq 0$,

$$
v_{\Delta}(x, y) \geq\|y\| /\|x\| \text {. }
$$

From the equivalences

$$
\begin{gathered}
1 \in \sigma(\Delta M) \Leftrightarrow \Delta(M v)=v \text { for some } v \text { with }\|v\|=1, \\
\operatorname{det}(M-\Delta)=0 \Leftrightarrow \Delta v=M v \text { for some } v \text { with }\|v\|=1, \\
1 \in \sigma(\Delta M) \Leftrightarrow \Delta(M v)=v \text { for some } v \text { with }\|v\|=1, \\
\operatorname{det}(M-\Delta)=0 \Leftrightarrow \Delta v=M v \text { for some } v \text { with }\|v\|=1,
\end{gathered}
$$

it follows that

$$
\begin{aligned}
& \mu_{\Delta}(M)=\left(\inf \left\{v_{\Delta}(M v, v) ; v \in \mathbb{C}^{n},\|v\|=1\right\}\right)^{-1}, \\
& \tilde{\mu}_{\Delta}(M)=\inf \left\{v_{\Delta}(v, M v) ; v \in \mathbb{C}^{n},\|v\|=1\right\} .
\end{aligned}
$$

Let $\Delta \in \operatorname{Herm}(n)$. Then the relation $\Delta x=y$ implies that $\mathfrak{I}\left(x^{*} y\right)=\mathfrak{I}\left(x^{*} \Delta x\right)=0$. Suppose that $\mathfrak{I}\left(x^{*} y\right)=0$ and $x \neq 0$. Then by [18, Theorem A.2] there exists $\Delta \in \operatorname{Herm}(n)$ with $\Delta x=y$ and $\|\Delta\|=\|y\| /\|x\|$. This combined with (2.4) yields that, for any $x, y \in \mathbb{C}^{n}, x \neq 0$,

$$
v_{\text {Herm }}(x, y)= \begin{cases}\|y\| /\|x\| & \text { if } x \neq 0 \quad \text { and } \quad \mathfrak{J}\left(x^{*} y\right)=0 \\ 0 & \text { if } x=y=0 \\ \infty & \text { otherwise. }\end{cases}
$$

For any $v \in \mathbb{C}^{n}$, we have that $\mathfrak{I}\left(v^{*}(M v)\right)=(1 / 2 i)\left(v^{*}(M v)-(M v)^{*} v\right)=$ $-(1 / 2) v^{*} M_{h} v$. Hence, (2.5) combined with (2.6) yields

$$
\begin{aligned}
& \mu_{\text {Herm }}(M)=\sup \left\{\|M v\| ; v \in \mathbb{C}^{n},\|v\|=1, v^{*} M_{h} v=0\right\}, \\
& \tilde{\mu}_{\text {Herm }}(M)=\inf \left\{\|M v\| ; v \in \mathbb{C}^{n},\|v\|=1, v^{*} M_{h} v=0\right\} .
\end{aligned}
$$

Suppose that $M_{h}$ is definite. Then $v^{*} M_{h} v \neq 0$ for all $v \neq 0$. Hence, $\mu_{\text {Herm }}(M)=0$ and $\tilde{\mu}_{\text {Herm }}(M)=\infty$. Suppose $M_{h}$ is not definite. Then the set of unit vectors $v$ satisfying $v^{*} M_{h} v=0$ is nonempty and closed. This concludes the proof of claim (a).

Let $\Delta \in \operatorname{Skew}(n)$. Then the relation $\Delta x=y$ implies that $x^{\top} y=x^{\top} \Delta x=0$. Suppose that $x^{\top} y=0$ and $x \neq 0$. Then by [18, Theorem A.2] there exists $\Delta \in \operatorname{Skew}(n)$ with $\Delta x=y$ and $\|\Delta\|=\|y\| /\|x\|$. This combined with (2.4) yields that, for any $x, y \in \mathbb{C}^{n}$, $x \neq 0$,

$$
v_{\text {Skew }}(x, y)= \begin{cases}\|y\| /\|x\| & \text { if } x \neq 0 \quad \text { and } \quad y^{T} x=0 \\ 0 & \text { if } x=y=0 \\ \infty & \text { otherwise. }\end{cases}
$$

Copyright ( by SIAM. Unauthorized reproduction of this article is prohibited. 
We have, for any $v \in \mathbb{C}^{n}$, that $(M v)^{\top} v=(1 / 2)\left((M v)^{\top} v+v^{\top}(M v)\right)=(1 / 2) v^{\top} M_{s} v$. Furthermore, if $n \geq 2$, then by Lemma 6.2 there exists a unit vector $v$ with $v^{\top} M_{s} v=0$. Hence, (2.7) combined with (2.5) yields claim (b).

If $x \neq 0$, then by [18, Theorem A.2] there exists $\Delta \in \operatorname{Sym}(n)$ such that $\Delta x=y$ and $\|\Delta\|=\|y\| /\|x\|$. Hence, we have

$$
v_{\text {Sym }}(x, y)= \begin{cases}\|y\| /\|x\| & \text { if } x \neq 0, \\ 0 & \text { if } x=y=0 \\ \infty & \text { otherwise. }\end{cases}
$$

Now (2.5) yields claim (c).

3. Computation of $\boldsymbol{\mu}$ for the Hermitian and the skew-symmetric case. Based on the identities (2.2) and (2.3) one can derive the following two theorems. They provide computable formulas for the $\mu$-values with respect to Hermitian and skewsymmetric perturbations. In order not to disturb the flow of exposition, the proofs are given in section 6 .

Theorem 3.1. Let $M \in \mathbb{C}^{n \times n}, M_{h}=i\left(M-M^{*}\right)$, and

$$
\begin{aligned}
& \phi(t)=\lambda_{\max }\left(M^{*} M+t M_{h}\right), \\
& \tilde{\phi}(t)=\lambda_{\min }\left(M^{*} M+t M_{h}\right), \quad t \in \mathbb{R},
\end{aligned}
$$

where $\lambda_{\max }$ and $\lambda_{\min }$ denote the largest and the smallest eigenvalue, respectively. Then the function $\phi$ is convex and the function $\tilde{\phi}$ is concave. Suppose that $M_{h}$ is not definite. Then

$$
\mu_{\text {Herm }}(M)=\left(\inf _{t \in \mathbb{R}} \phi(t)\right)^{1 / 2}, \quad \tilde{\mu}_{\text {Herm }}(M)=\left(\sup _{t \in \mathbb{R}} \tilde{\phi}(t)\right)^{1 / 2} .
$$

Furthermore, the following statements hold.

(i) If $M_{h}$ is indefinite, then the infimum is attained in the interval $\left[t_{1}, t_{2}\right]$ and the supremum is attained in the interval $\left[-t_{2},-t_{1}\right]$, where

$$
t_{1}=\frac{\sigma_{\max }^{2}(M)-\sigma_{\min }^{2}(M)}{\lambda_{\min }\left(M_{h}\right)}, \quad t_{2}=\frac{\sigma_{\max }^{2}(M)-\sigma_{\min }^{2}(M)}{\lambda_{\max }\left(M_{h}\right)} .
$$

(ii) Suppose $M_{h}$ is positive (negative) semidefinite but not definite. Then the functions $\phi: \mathbb{R} \rightarrow \mathbb{R}$ and $\tilde{\phi}: \mathbb{R} \rightarrow \mathbb{R}$ are both increasing (both decreasing). Moreover, we have

$$
\begin{aligned}
\mu_{\mathrm{Herm}}(M) & =\sigma_{\max }(M V) \\
& = \begin{cases}\left(\lim _{t \rightarrow-\infty} \phi(t)\right)^{1 / 2} & \text { if } M_{h} \text { is positive semidefinite, } \\
\left(\lim _{t \rightarrow \infty} \phi(t)\right)^{1 / 2} & \text { if } M_{h} \text { is negative semidefinite, }\end{cases} \\
\tilde{\mu}_{\mathrm{Herm}}(M) & =\sigma_{\min }(M V) \\
& = \begin{cases}\left(\lim _{t \rightarrow \infty} \tilde{\phi}(t)\right)^{1 / 2} & \text { if } M_{h} \text { is positive semidefinite, } \\
\left(\lim _{t \rightarrow-\infty} \tilde{\phi}(t)\right)^{1 / 2} & \text { if } M_{h} \text { is negative semidefinite, }\end{cases}
\end{aligned}
$$

where $V$ is any matrix whose columns form an orthonormal basis of ker $M_{h}$. Remark 3.2. For any $M \in \mathbb{C}^{n \times n}$ and any $t \in \mathbb{R}$,

$$
M^{*} M+t M_{h}=(M-i t I)^{*}(M-i t I)-t^{2} I .
$$

Copyright @ ( by SIAM. Unauthorized reproduction of this article is prohibited. 
Hence, the objective functions in Theorem 3.1 can be written as

$$
\phi(t)=\sigma_{\max }^{2}(M-i t I)-t^{2}, \quad \tilde{\phi}(t)=\sigma_{\min }^{2}(M-i t I)-t^{2} .
$$

Let $\mathcal{I} \subseteq \mathbb{R}$ be an interval. A function $f: \mathcal{I} \rightarrow \mathbb{R}$ is said to be unimodal ${ }^{2}$ if each local extremum of $f$ attained in the interior of $\mathcal{I}$ equals the global minimum of $f$. The global minimum of a continuous unimodal function on a compact interval $\mathcal{I}$ can reliably be found by golden section search [21, section 2.1] or by any other locally minimizing algorithm. The functions $\phi$ and $-\tilde{\phi}$, being convex, are unimodal. In [13] we proposed a bisection method using the derivative of $\phi$ to compute $\mu_{\mathrm{Herm}}(M)=\min _{t \in\left[t_{1}, t_{2}\right]} \phi(t)$ in the case that $M_{h}$ is indefinite.

Next, we consider skew-symmetric perturbations.

Theorem 3.3. Let $M \in \mathbb{C}^{n \times n}, n \geq 2$, and let $M_{s}=M+M^{\top}$. For $t \in[0, \infty)$, let

$$
F(t)=\left[\begin{array}{cc}
M^{*} M & t \bar{M}_{s} \\
t M_{s} & \bar{M}^{*} M
\end{array}\right] \in \operatorname{Herm}(2 n)
$$

and

$$
\psi(t)=\lambda_{2}(F(t)), \quad \tilde{\psi}(t)=\lambda_{2 n-1}(F(t)),
$$

where $\lambda_{2}$ and $\lambda_{2 n-1}$ denote the second largest and the second smallest eigenvalue, respectively. Then the functions $\psi(\cdot)$ and $-\tilde{\psi}(\cdot)$ are both unimodal on $[0, \infty)$, and

$$
\begin{aligned}
\mu_{\text {Skew }}(M) & =\left(\inf _{t \in[0, \infty)} \psi(t)\right)^{1 / 2}, \\
\tilde{\mu}_{\text {Skew }}(M) & =\left(\sup _{t \in[0, \infty)} \tilde{\psi}(t)\right)^{1 / 2} .
\end{aligned}
$$

Furthermore, the following statements hold.

(i) If $\operatorname{rank}\left(M_{s}\right) \geq 2$, then both the infimum and the supremum are attained in the interval $\left[0, t_{1}\right]$, where $t_{1}=2 \sigma_{\max }^{2}(M) / \sigma_{2}\left(M_{s}\right)$ and $\sigma_{2}(\cdot)$ denotes the second largest singular value.

(ii) Suppose $\operatorname{rank}\left(M_{s}\right)=1$. Then the function $\psi:[0, \infty) \rightarrow \mathbb{R}$ is decreasing, the function $\tilde{\psi}:[0, \infty) \rightarrow \mathbb{R}$ is increasing, and

$$
\begin{aligned}
& \mu_{\text {Skew }}(M)=\sigma_{\max }(M V)=\lim _{t \rightarrow \infty} \psi(t), \\
& \tilde{\mu}_{\text {Skew }}(M)=\sigma_{\min }(M V)=\lim _{t \rightarrow \infty} \tilde{\psi}(t),
\end{aligned}
$$

where $V$ is any matrix whose columns form an orthonormal basis of ker $M_{s}$.

(iii) If $M_{s}=0$, then the functions $\psi(\cdot)$ and $\tilde{\psi}(\cdot)$ are both constant, and

$$
\begin{aligned}
& \mu_{\text {Skew }}(M)=\sigma_{\max }(M)=\psi(0), \\
& \tilde{\mu}_{\text {Skew }}(M)=\sigma_{\min }(M)=\tilde{\psi}(0) .
\end{aligned}
$$

Remark 3.4. The pencil $F(t)$ in Theorem 3.3 satisfies

$$
F(t)=\left[\begin{array}{cc}
M & t I \\
t I & \bar{M}
\end{array}\right]^{*}\left[\begin{array}{cc}
M & t I \\
t I & \bar{M}
\end{array}\right]-t^{2} I
$$

\footnotetext{
${ }^{2}$ The definition of unimodality is not unique in the literature. By our definition strictly monotone functions
} as well as constant functions are unimodal.

Copyright ( by SIAM. Unauthorized reproduction of this article is prohibited. 
Thus, the objective functions in Theorem 3.3 can be written as

$$
\psi(t)=\sigma_{2}^{2}\left(\left[\begin{array}{cc}
M & t I \\
t I & \bar{M}
\end{array}\right]\right)-t^{2}, \quad \tilde{\psi}(t)=\sigma_{2 n-1}^{2}\left(\left[\begin{array}{cc}
M & t I \\
t I & \bar{M}
\end{array}\right]\right)-t^{2},
$$

where $\sigma_{2}$ and $\sigma_{2 n-1}$ denote the second largest and the second smallest singular value, respectively. For the computation of $\psi$ using the representation (3.2), see [13].

Example 3.5. We compute $\mu_{\text {Skew }}(M)$ and $\tilde{\mu}_{\text {Skew }}(M)$ for $M=i I-A$, where

$$
A=\left[\begin{array}{ccc}
0 & 10 & 0 \\
-10 & 0 & 0 \\
0 & 0 & 0
\end{array}\right]
$$

In this case the pencil $F(t)$ satisfies

$$
F(t)=\left[\begin{array}{cc}
M^{*} M & t \bar{M}_{s} \\
t M_{s} & M^{*} M
\end{array}\right]=\left[\begin{array}{cc}
H & -2 i t I \\
2 i t I & \bar{H}
\end{array}\right], \quad H:=M^{*} M=\left[\begin{array}{ccc}
101 & 20 i & 0 \\
-20 i & 101 & 0 \\
0 & 0 & 1
\end{array}\right] .
$$

For $\lambda \in \mathbb{C} \backslash \sigma(H)$, we have $\lambda I-F(t)=T G(\lambda) T^{*}$, where

$$
G(\lambda)=\left[\begin{array}{cc}
\lambda I-H & 0 \\
0 & (\lambda I-\bar{H})-4 t^{2}(\lambda I-H)^{-1}
\end{array}\right], \quad T=\left[\begin{array}{cc}
I & 0 \\
2 i t(\lambda I-H)^{-1} & I
\end{array}\right] .
$$

Thus, the characteristic polynomial of $F(t)$ is

$$
\begin{aligned}
\operatorname{det}(\lambda I-F(t)) & =\operatorname{det}(G(\lambda)) \\
& =\operatorname{det}\left((\lambda I-H)(\lambda I-\bar{H})-4 t^{2} I\right) \\
& =\left(\lambda^{2}-2 \lambda+1-4 t^{2}\right)\left(\lambda^{2}-202 \lambda+9801-4 t^{2}\right)^{2} .
\end{aligned}
$$

Hence, the six eigenvalues of $F(t)$ (denoted by $\left.\ell_{k}(t)\right)$ are

$$
\begin{aligned}
& \ell_{1}(t)=1+2 t, \\
& \ell_{2}(t)=1-2 t, \\
& \ell_{3}(t)=\ell_{4}(t)=101+2 \sqrt{100+t^{2}}, \\
& \ell_{5}(t)=\ell_{6}(t)=101-2 \sqrt{100+t^{2}} .
\end{aligned}
$$

The eigenvalue curves $\ell_{k}(\cdot)$ are displayed in Figure 3.1. The second largest eigenvalue of $F(t)$ is $\psi(t)=\lambda_{2}(F(t))=\ell_{3}(t)=\ell_{4}(t)$. The function $\psi(t)$ attains its minimum at $t=0$. Thus, $\mu_{\text {Skew }}(M)=\sqrt{\psi(0)}=11$. The second smallest eigenvalue of $F(t)$ is $\tilde{\psi}(t)=$ $\lambda_{5}(F(t))=\min \left\{\ell_{1}(t), \ell_{5}(t)\right\}$. The function $\tilde{\psi}(t)$ attains its maximum at $t=24$, where the curves $\ell_{1}(\cdot)$ and $\ell_{5}(\cdot)$ meet. Thus, $\tilde{\mu}_{\text {Skew }}(M)=\sqrt{\tilde{\psi}(24)}=7$. The skew-symmetric spectral value sets

$$
\sigma_{\text {Skew }}(A, \delta)=\bigcup_{\Delta \in \operatorname{Skew}(3),\|\Delta\|<\delta} \sigma(A+\Delta)=\left\{s \in \mathbb{C} ; \tilde{\mu}_{\text {Skew }}(s I-A)<\delta\right\}, \quad \delta \in\{4,7,9\},
$$

Copyright () by SIAM. Unauthorized reproduction of this article is prohibited. 

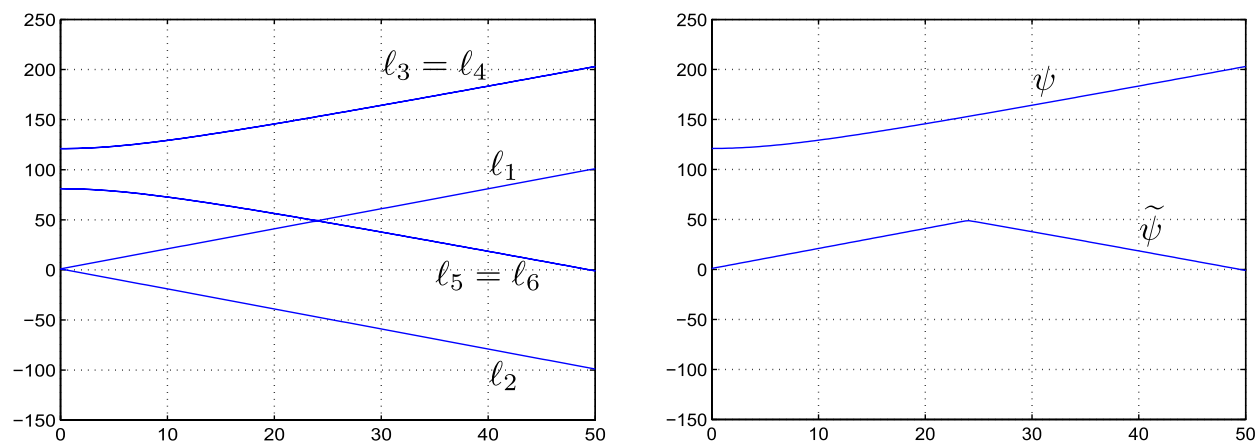

FIG. 3.1. The eigenvalue curves $\ell_{k}(\cdot), \psi(\cdot)$, and $\tilde{\psi}(\cdot)$ from Example 3.5.

are depicted in the upper row of Figure 3.2. The lower row shows the unstructured spectral value sets

$$
\sigma_{\mathbb{C}^{3 \times 3}}(A, \delta)=\bigcup_{\Delta \in \mathbb{C}^{3 \times 3},\|\Delta\|<\delta} \sigma(A+\Delta)=\left\{s \in \mathbb{C} ; \sigma_{\min }(s I-A)<\delta\right\}, \quad \delta \in\{4,7,9\} .
$$

The crosses mark the eigenvalues of $A$. Observe that the eigenvalue 0 is an isolated point of $\sigma_{\text {Skew }}(A, \delta), \delta=4,7$. This can be explained as follows. Let $D$ be a disk about 0 that does not contain an eigenvalue of $A$ different from 0 . Let $\Delta \in \operatorname{Skew}(3)$. If $\delta>0$ is sufficiently small and $\|\Delta\|<\delta$, then by continuity only one eigenvalue of $A+\Delta$ is contained in $D$. This eigenvalue is 0 since $A+\Delta$ is a skew-symmetric matrix of odd dimension.

$\delta=4$
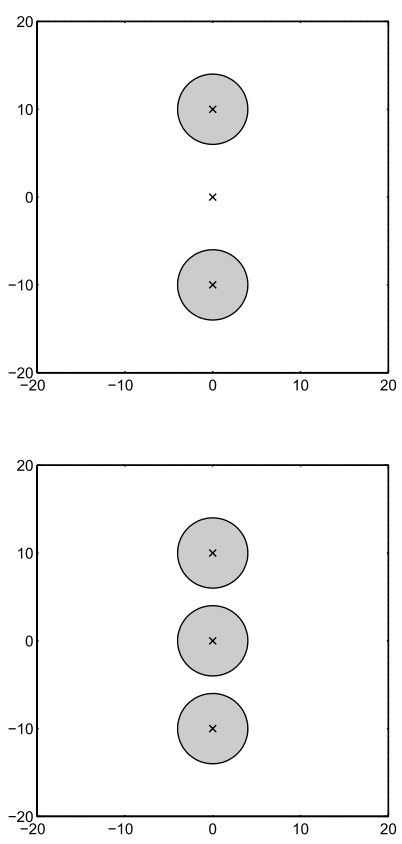

$\delta=7$
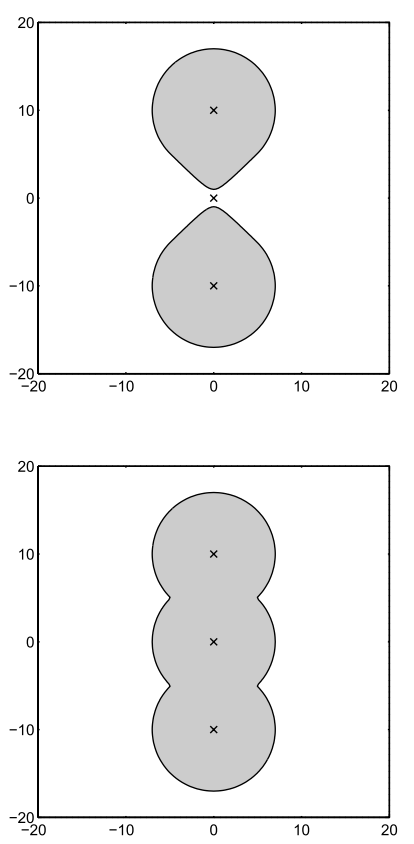

$\delta=9$
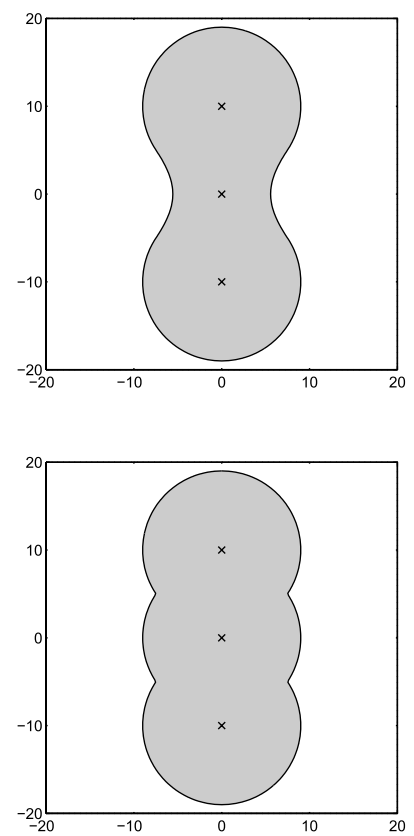

FIG. 3.2. The sets $\sigma_{\text {Skew }}(A, \delta)$ (upper row) and $\sigma_{\mathbb{C}^{3 \times 3}}(A, \delta)$ (lower row) for $A$ defined in (3.3).

Copyright @ by SIAM. Unauthorized reproduction of this article is prohibited. 
4. Self- and skew-adjoint matrices. We now treat $\mu$-values with respect to linear subspaces which are induced by a scalar product on $\mathbb{C}^{n}$. Specifically we show that these $\mu$-values are closely related to the $\mu$-values with respect to Hermitian, symmetric, and skew-symmetric perturbations. For nonsingular $\Pi \in \mathbb{C}^{n \times n}$, we consider the scalar products

$$
\langle x, y\rangle_{\Pi}=x^{\star} \Pi y, \quad x, y \in \mathbb{C}^{n}, \quad \star \in\{*, \top\} .
$$

Depending on whether $\star=\top$ or $\star=*$ the scalar product is a bilinear form or a sesquilinear form. We assume that $\Pi$ satisfies a symmetry relation of the form

$$
\Pi^{\star}=\epsilon_{0} \Pi, \quad \text { with } \epsilon_{0}=-1 \quad \text { or } \quad \epsilon_{0}=1 .
$$

A matrix $\Delta \in \mathbb{C}^{n \times n}$ is said to be self-adjoint (skew-adjoint) with respect to the scalar product $\langle\cdot, \cdot\rangle_{\Pi}$ if

$$
\langle\Delta x, y\rangle_{\Pi}=\epsilon\langle x, \Delta y\rangle_{\Pi} \quad \text { for all } x, y \in \mathbb{C}^{n}
$$

and $\epsilon=1 \quad(\epsilon=-1)$. It is easy to see that the relation (4.2) is equivalent to $(\Pi \Delta)^{\star}=\epsilon_{0} \epsilon \Pi \Delta$. We denote the sets of self- and skew-adjoint matrices by

$$
\operatorname{struct}(\Pi, \star, \epsilon):=\left\{\Delta \in \mathbb{C}^{n \times n} ;(\Pi \Delta)^{\star}=\epsilon_{0} \epsilon \Pi \Delta\right\} .
$$

Thus,

$$
\Delta \in \operatorname{struct}(\Pi, \star, \epsilon) \Leftrightarrow \begin{cases}\Pi \Delta \in \operatorname{Herm}(n) & \text { if } \epsilon_{0} \epsilon=1, \quad \star=*, \\ \Pi \Delta \in \operatorname{Sym}(n) & \text { if } \epsilon_{0} \epsilon=1, \quad=\top, \\ \Pi \Delta \in \operatorname{Skew}(n) & \text { if } \epsilon_{0} \epsilon=-1, \quad \star=\top, \\ \pm i \Pi \Delta \in \operatorname{Herm}(n) & \text { if } \epsilon_{0} \epsilon=-1, \quad \star=* .\end{cases}
$$

In many applications $\Pi$ is unitary. The most common examples are $\Pi \in\left\{\operatorname{diag}\left(I_{k}\right.\right.$, $\left.\left.-I_{n-k}\right), E_{n}, J_{n}\right\}$, where

$$
J_{n}:=\left[\begin{array}{cc}
0 & I_{n} \\
-I_{n} & 0
\end{array}\right] \in \mathbb{C}^{2 n \times 2 n}, \quad E_{n}:=\left[\begin{array}{l}
. \\
1
\end{array}\right] \in \mathbb{C}^{n \times n} .
$$

For unitary $\Pi$ the $\mu$-values of the associated self- and skew-adjoint classes can be expressed in terms of the $\mu$-values for $\operatorname{Herm}(n), \operatorname{Sym}(n)$, and $\operatorname{Skew}(n)$.

Proposition 4.1. Suppose $\Pi \in \mathbb{C}^{n \times n}$ is unitary and satisfies $\Pi^{\star}=\epsilon_{0} \Pi$ with $\epsilon_{0}=-1$ or $\epsilon_{0}=1$. Let struct $=\operatorname{struct}(\Pi, \star, \epsilon)$. Then for any $M \in \mathbb{C}^{n \times n}$,

$$
\mu_{\text {struct }}(M)= \begin{cases}\mu_{\mathrm{Herm}}\left(M \Pi^{*}\right) & \text { if } \epsilon_{0} \epsilon=1, \quad \star=*, \\ \mu_{\text {Sym }}\left(M \Pi^{*}\right) & \text { if } \epsilon_{0} \epsilon=1, \quad \star=\top, \\ \mu_{\text {Skew }}\left(M \Pi^{*}\right) & \text { if } \epsilon_{0} \epsilon=-1, \quad \star=\top, \\ \mu_{\text {Herm }}\left( \pm i M \Pi^{*}\right) & \text { if } \epsilon_{0} \epsilon=-1, \quad \star=*,\end{cases}
$$

and

Copyright ( by SIAM. Unauthorized reproduction of this article is prohibited. 


$$
\tilde{\mu}_{\text {struct }}(M)= \begin{cases}\tilde{\mu}_{\text {Herm }}(\Pi M) & \text { if } \epsilon_{0} \epsilon=1, \quad \star=*, \\ \tilde{\mu}_{\text {Sym }}(\Pi M) & \text { if } \epsilon_{0} \epsilon=1, \quad \star=\top, \\ \tilde{\mu}_{\text {Skew }}(\Pi M) & \text { if } \epsilon_{0} \epsilon=-1, \quad \star=\top, \\ \tilde{\mu}_{\text {Herm }}( \pm i \Pi M) & \text { if } \epsilon_{0} \epsilon=-1, \quad \star=* .\end{cases}
$$

Proof. Since $\Pi$ is unitary we have

$$
\begin{aligned}
\mu_{\text {struct }}(M) & =(\inf \{\|\Delta\| ; \Delta \in \text { struct, } 1 \in \sigma(\Delta M)\})^{-1} \\
& =\left(\inf \left\{\|\Pi \Delta\| ; \Delta \in \text { struct, } 1 \in \sigma\left((\Pi \Delta)\left(M \Pi^{*}\right)\right)\right\}\right)^{-1}, \\
\tilde{\mu}_{\text {struct }}(M) & =\inf \{\|\Delta\| ; \Delta \in \text { struct, } \operatorname{det}(M-\Delta)=0\} \\
& =\inf \{\|\Pi \Delta\| ; \Delta \in \operatorname{struct}, \operatorname{det}(\Pi M-\Pi \Delta)=0\} .
\end{aligned}
$$

Thus, the first three identities in (4.5) and (4.6) are consequences of the first three equivalences of (4.3). On replacing in (4.7) and (4.8) $\Pi$ by $\pm i \Pi$ one obtains the fourth identity in (4.5) and (4.6) from the fourth equivalence of (4.3).

5. Application: Spectral value sets for Hamiltonian matrices. A matrix which is skew-adjoint with respect to the sesquilinear form induced by $J_{n}$ is called Hamiltonian. Let $\operatorname{Ham}(n):=\left\{\Delta \in \mathbb{C}^{2 n \times 2 n} ; \Delta^{*} J_{n}=-J_{n} \Delta\right\}$ denote the set of complex Hamiltonian matrices. Each $H \in \operatorname{Ham}(n)$ has block structure

$$
H=\left[\begin{array}{cc}
A & C \\
B & -A^{*}
\end{array}\right] \quad \text { with } A \in \mathbb{C}^{n \times n} \quad \text { and } \quad B, C \in \operatorname{Herm}(n) .
$$

The spectral value sets of $H$ with respect to Hamiltonian perturbations are by (1.11)

$$
\sigma_{\text {Ham }}(H, \delta)=\bigcup_{\Delta \in \operatorname{Ham}(n),\|\Delta\|<\delta} \sigma(H+\Delta)=\{s \in \mathbb{C} ; f(s)<\delta\}, \quad \delta>0,
$$

where $f(s):=\tilde{\mu}_{\mathrm{Ham}}(s I-H), s \in \mathbb{C}$. Let $\Phi(s):=J_{n}(s I-H)=\left[\begin{array}{cc}-B & s I+A^{*} \\ -s I+A & C\end{array}\right]$ and $\Phi_{h}(s):=i\left(\Phi(s)-\Phi(s)^{*}\right)=2 i(\Re s) J_{n}$. Then by Corollary 4.1 and Theorem 2.1

$$
\begin{aligned}
f(s) & =\tilde{\mu}_{\mathrm{Herm}}(\Phi(s)) \\
& =\min \left\{\|\Phi(s) v\| ; v \in \mathbb{C}^{2 n},\|v\|=1, v^{*} \Phi_{h}(s) v=0\right\} \\
& = \begin{cases}\sigma_{\min }(s I-H) & \text { if } s \in i \mathbb{R}, \\
\min \left\{\|(s I-H) v\| ; v \in \mathbb{C}^{2 n},\|v\|=1, v^{*} J_{n} v=0\right\} & \text { otherwise. }\end{cases}
\end{aligned}
$$

The latter equation holds since $\Phi(s)=0$ iff $s \in i \mathbb{R}$, and $\|\Phi(s) v\|=\|(s I-H) v\|$ for all $v \in \mathbb{C}^{2 n}$. Since $\tilde{\mu}_{\mathbb{C}^{2 n \times 2 n}}(s I-H)=\sigma_{\min }(s I-H),(5.2)$ implies

$$
\sigma_{\mathrm{Ham}}(H) \cap(i \mathbb{R})=\sigma_{\mathbb{C}^{2 n \times 2 n}}(H) \cap(i \mathbb{R}) .
$$

Let $s \in \mathbb{C} \backslash(i \mathbb{R})$. Then $\Phi_{h}(s)$ is indefinite since $\lambda_{\max }\left(\Phi_{h}(s)\right)=-\lambda_{\min }\left(\Phi_{h}(s)\right)=2|\Re s|$. Thus, by Theorem 3.1 


$$
\begin{aligned}
f(s) & =\left(\max _{t \in\left[-\frac{t_{0}}{2\left|\Re_{s}\right|} \cdot \frac{t_{0} \mid \boldsymbol{R}_{s} s}{}\right]} \lambda_{\min }\left(\Phi(s)^{*} \Phi(s)+t \Phi_{h}(s)\right)\right)^{\frac{1}{2}} \\
& =\left(\max _{\tau \in\left[-t_{0}, t_{0}\right]} \lambda_{\min }\left((s I-H)^{*}(s I-H)+\tau i J_{n}\right)\right)^{\frac{1}{2}},
\end{aligned}
$$

where $t_{0}=\sigma_{\max }^{2}(s I-H)-\sigma_{\min }^{2}(s I-H)$. Formula (5.3) and the upper equation in (5.2) have been used to compute the spectral value sets $\sigma_{\mathrm{Ham}}\left(H_{\gamma}, 1\right)$ in the upper row of Figure 5.1. Here,

$$
H_{\gamma}=\left[\begin{array}{cc}
0 & -\gamma^{-1} B \\
\gamma B & 0
\end{array}\right], \quad B=\operatorname{diag}(1,6,-6), \quad \gamma \in\{1,1.3,5,6\} .
$$

The lower row in the figure shows the sets $\sigma_{\mathbb{C}^{6 \times 6}}\left(H_{\gamma}, 1\right)$ for comparison. The crosses mark the eigenvalues of $H_{\gamma}$. The pictures illustrate the fact that spectral value sets of Hamiltonian matrices with respect to Hamiltonian perturbations are not necessarily open. The proposition below states basic topological facts about these sets.

Proposition 5.1. Let $H \in \operatorname{Ham}(n)$ and $\delta>0$. Then

(a) $\sigma_{\mathrm{Ham}}(H, \delta) \cap(i \mathbb{R})$ is an open subset of $i \mathbb{R}$.

(b) $\sigma_{\text {Ham }}(H, \delta) \backslash(i \mathbb{R})$ is an open subset of $\mathbb{C}$.

Let $s_{0} \in i \mathbb{R}$ be a purely imaginary eigenvalue of $H$, and let $\mathcal{E}$ denote the associated eigenspace.

(c) If there exists a $v \in \mathcal{E} \backslash\{0\}$ such that $v^{*} J_{n} v=0$, then $s_{0}$ is an interior point of $\sigma_{\mathrm{Ham}}(H, \delta)$.

(d) Suppose that $v^{*} J_{n} v \neq 0$ for all $v \in \mathcal{E} \backslash\{0\}$. Then there exists a $\delta_{0}>0$ and a disk $D$ with center $s_{0}$ such that $\sigma_{\mathrm{Ham}}(H, \delta) \cap D \subset i \mathbb{R}$ for all $\delta<\delta_{0}$.
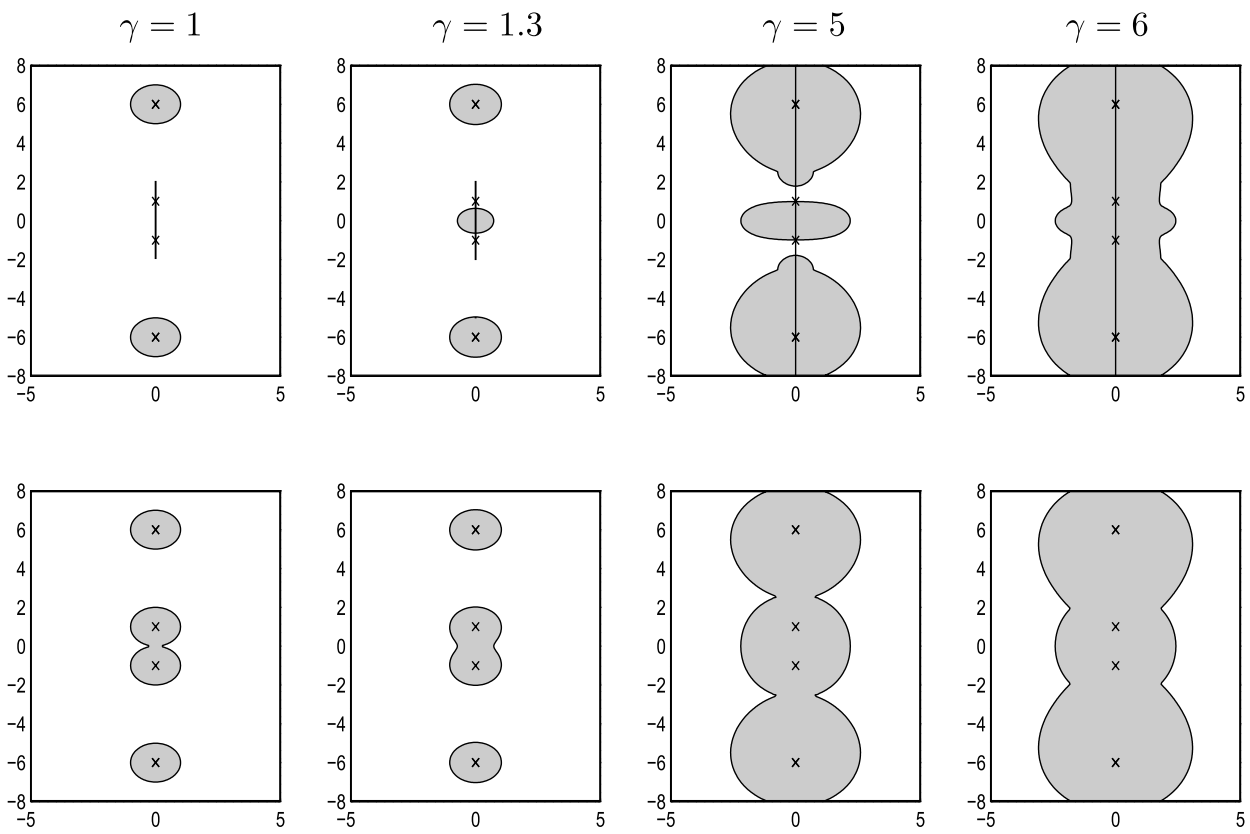

FIG. 5.1. The sets $\sigma_{\text {Ham }}\left(H_{\gamma}, 1\right)$ (upper row) and $\sigma_{\mathbb{C}^{6 \times 6}}\left(H_{\gamma}, 1\right)$ (lower row). 
Proof. For $s \in \mathbb{C}$, let $f_{1}(s)=\sigma_{\min }(s I-H)$ and $f_{2}(s)=\min _{s \in K}\|(s I-H) v\|$, where $K=\left\{v \in \mathbb{C}^{2 n} ;\|v\|=1, v^{*} J_{n} v=0\right\}$. Then $f_{1}$ and $f_{2}$ are continuous at all $s \in \mathbb{C}$. (The continuity of $f_{2}$ follows from the continuity of the map $(s, v) \mapsto\|(s I-H) v\|$ and the compactness of $K$.) Furthermore, by (5.2)

$$
\begin{aligned}
\sigma_{\text {Ham }}(H, \delta) \cap(i \mathbb{R}) & =\left\{s \in i \mathbb{R} ; f_{1}(s)<\delta\right\}, \\
\sigma_{\text {Ham }}(H, \delta) \backslash(i \mathbb{R}) & =\left\{s \in \mathbb{C} \backslash i \mathbb{R} ; f_{2}(s)<\delta\right\} .
\end{aligned}
$$

Hence, $\sigma_{\text {Ham }}(H, \delta) \cap(i \mathbb{R})$ is an open subset of $i \mathbb{R}$ and $\sigma_{\text {Ham }}(H, \delta) \backslash(i \mathbb{R})$ is an open subset of $\mathbb{C}$. Now let $s_{0} \in i \mathbb{R}$ be a purely imaginary eigenvalue of $H$. Then $f_{1}\left(s_{0}\right)=0$. Suppose there exists an eigenvector $v \neq 0$ with $v^{*} J_{n} v=0$. Then $f_{2}\left(s_{0}\right)=0$. From (5.4) it then follows that $s_{0}$ is an interior point of $\sigma_{\mathrm{Ham}}(H, \delta)$. Assume there is no eigenvector such that $v^{*} J_{n} v=0$. Then $f_{2}\left(s_{0}\right)>0$. Let $\delta_{0}=f_{2}\left(s_{0}\right) / 2$. Then by continuity there is a disk $D$ with center $s_{0}$ such that $f_{2}(s) \geq \delta_{0}$ for $s \in D$. Thus, by (5.4)

$$
\left(\sigma_{\text {Ham }}(H, \delta) \backslash(i \mathbb{R})\right) \cap D=\varnothing \text { for } \delta<\delta_{0} .
$$

6. Proofs of Theorems 3.1 and 3.3. In what follows $\lambda_{\max }(H)=\lambda_{1}(H) \geq \lambda_{2}(H) \geq$ $\ldots \geq \lambda_{n}(H)=\lambda_{\min }(H)$ denote the eigenvalues of $H \in \operatorname{Herm}(n)$ in decreasing order. The corresponding eigenspaces are denoted by $\mathcal{E}_{k}(H), k=1, \ldots, n$. Recall that

$$
\lambda_{n+1-k}(H)=-\lambda_{k}(-H)
$$

and

$$
\lambda_{k}(H)+\lambda_{\min }(G) \leq \lambda_{k}(H+G) \leq \lambda_{k}(H)+\lambda_{\max }(G)
$$

for all $H, G \in \operatorname{Herm}(n), k=1, \ldots, n$. The proofs of Theorem 3.1 and 3.3 use the same technique as in [9], [17], [19], [22]. We need the following preliminary result on the eigenvalues and eigenvectors of a Hermitian pencil.

Proposition 6.1. Let $H_{0}, H_{1} \in \operatorname{Herm}(n)$ and $H(t)=H_{0}+t H_{1}, \quad t \in \mathbb{R}$, and $k \in\{1, \ldots, n\}$.

(a) Suppose the function $t \mapsto \lambda_{k}(H(t)), t \in \mathbb{R}$, attains a local extremum at $t_{0}$. Then there exists a unit vector $v \in \mathcal{E}_{k}\left(H\left(t_{0}\right)\right)$ such that $v^{*} H_{1} v=0$. Suppose the extremum is a local minimum and we have $k=n$ or $\lambda_{k}\left(H\left(t_{0}\right)\right)>\lambda_{k+1}\left(H\left(t_{0}\right)\right)$. Then $v^{*} H_{1} w=0$ for all $v, w \in \mathcal{E}_{k}\left(H\left(t_{0}\right)\right)$.

(b) Suppose $\lambda_{k}\left(H_{1}\right)=0$ and either $k=1$ or $\lambda_{k-1}\left(H_{1}\right)>0$. Then

$$
\lim _{t \rightarrow \infty} \lambda_{k}(H(t))=\lambda_{\max }\left(V^{*} H_{0} V\right),
$$

where $V \in \mathbb{C}^{n \times p}$ is a matrix whose columns form an orthonormal basis of ker $H_{1}$.

Proof. By [16, Thm. II.1.10 and subsec. II.4.5] there exist analytic functions $\ell_{j}: \mathbb{R} \rightarrow \mathbb{R}, v_{j}: \mathbb{R} \rightarrow \mathbb{R}^{n}, j=1, \ldots, n$, such that $H(t) v_{j}(t)=\ell_{j}(t) v_{j}(t)$ and

$$
v_{i}(t)^{*} v_{j}(t)= \begin{cases}1 & \text { if } i=j, \\ 0 & \text { otherwise }\end{cases}
$$

for $i, j \in\{1, \ldots, n\}$ and $t \in \mathbb{R}$. Hence, the vectors $v_{j}(t)$ form an othonormal basis of eigenvectors of $H(t)$ and the numbers $\ell_{j}(t)$ are the corresponding eigenvalues not necessarily ordered up to size. By differentiating the identity $\ell_{j}(t) v_{i}(t)^{*} v_{j}(t)=$ $v_{i}(t)^{*} H(t) v_{j}(t), t \in \mathbb{R}$, we obtain

Copyright ( $\odot$ by SIAM. Unauthorized reproduction of this article is prohibited. 


$$
\begin{aligned}
\frac{d}{d t}\left(\ell_{j}(t) v_{i}(t)^{*} v_{j}(t)\right)= & \frac{d}{d t}\left(v_{i}(t)^{*} H(t) v_{j}(t)\right) \\
= & v_{i}(t)^{*} H^{\prime}(t) v_{j}(t)+v_{i}^{\prime}(t)^{*} H(t) v_{j}(t)+v_{i}(t)^{*} H(t) v_{j}^{\prime}(t) \\
= & v_{i}(t)^{*} H_{1} v_{j}(t)+\ell_{j}(t) v_{i}^{\prime}(t)^{*} v_{j}(t)+\ell_{i}(t) v_{i}(t)^{*} v_{j}^{\prime}(t) \\
= & v_{i}(t)^{*} H_{1} v_{j}(t) \\
& +\ell_{j}(t) \frac{d}{d t}\left(v_{i}(t)^{*} v_{j}(t)\right)+\left(\ell_{i}(t)-\ell_{j}(t)\right) v_{i}(t)^{*} v_{j}^{\prime}(t) .
\end{aligned}
$$

This combined with (6.4) yields the following facts.

Fact 1 . Let $i \neq j$ and $t \in \mathbb{R}$. If $\ell_{i}(t)=\ell_{j}(t)$, then $v_{i}(t)^{*} H_{1} v_{j}(t)=0$.

Fact 2. The derivative of $\ell_{j}(\cdot)$ at $t \in \mathbb{R}$ satisfies $\ell_{j}^{\prime}(t)=v_{j}(t)^{*} H_{1} v_{j}(t), j=1, \ldots, n$.

Let $\mathcal{J}_{k}(t)=\left\{j ; \ell_{j}(t)=\lambda_{k}(H(t))\right\}$. Then the vectors $v_{j}(t), j \in \mathcal{J}_{k}(t)$, form an orthonormal basis of the eigenspace $\mathcal{E}_{k}(H(t))$. For any pair of indices $i, j$, the analytic functions $t \mapsto \ell_{i}(t), t \mapsto \ell_{j}(t)$, are either identical or their graphs meet in a discrete set of points. Thus, for any $t_{0} \in \mathbb{R}$, there are an $\epsilon>0$ and indices $j_{1}, j_{2} \in \mathcal{J}_{k}\left(t_{0}\right)$ such that

$$
\lambda_{k}(H(t))= \begin{cases}\ell_{j_{1}}(t) & \text { for } t \in\left[t_{0}, t_{0}+\epsilon\right] \\ \ell_{j_{2}}(t) & \text { for } t \in\left[t_{0}-\epsilon, t_{0}\right]\end{cases}
$$

Hence, if the function $t \mapsto \lambda_{k}(H(t))$ attains a local minimum at $t_{0}$, then $\ell_{j_{1}}^{\prime}\left(t_{0}\right)=$ $v_{j_{1}}\left(t_{0}\right)^{*} H_{1} v_{j_{1}}\left(t_{0}\right) \geq 0$ and $\ell_{j_{2}}^{\prime}\left(t_{0}\right)=v_{j_{2}}\left(t_{0}\right)^{*} H_{1} v_{j_{2}}\left(t_{0}\right) \leq 0$. Clearly, if $j_{1}=j_{2}$, then $v_{j_{1}}\left(t_{0}\right)^{*} H_{1} v_{j_{1}}\left(t_{0}\right)=0$. If $j_{1} \neq j_{2}$, then by continuity there exists a vector $v \in \mathcal{E}_{k}\left(H\left(t_{0}\right)\right) \backslash$ $\{0\}$ of the form $v=\cos (\alpha) v_{j_{1}}\left(t_{0}\right)+\sin (\alpha) v_{j_{2}}\left(t_{0}\right), \alpha \in[0, \pi / 2]$, such that $v^{*} H_{1} v=0$. An analogous argument holds if $\lambda_{k}(\cdot)$ attains a local maximum. Thus, we have shown the first statement of (a). To prove the second consider a $t_{0} \in \mathbb{R}$ such that $\lambda_{k}\left(H\left(t_{0}\right)\right)>$ $\lambda_{k+1}\left(H\left(t_{0}\right)\right)$. Then by continuity and the definition of $\mathcal{J}_{k}\left(t_{0}\right)$ there exists an $\epsilon>0$ such that

$$
\ell_{j}(t)>\lambda_{k+1}(H(t)) \quad \text { for all } j \in \mathcal{J}_{k}\left(t_{0}\right) \quad \text { and all } \quad t \in\left[t_{0}-\epsilon, t_{0}+\epsilon\right] .
$$

Since each $\ell_{j}(t)$ equals one of the eigenvalues of $H(t)$ it follows that

$$
\ell_{j}(t) \geq \lambda_{k}(H(t)) \quad \text { for all } j \in \mathcal{J}_{k}\left(t_{0}\right) \quad \text { and all } \quad t \in\left[t_{0}-\epsilon, t_{0}+\epsilon\right] .
$$

Note that the latter statement trivially holds for all $t_{0} \in \mathbb{R}$ if $k=n$. Suppose now that $\lambda_{k}$ attains a local minimum at $t_{0}$. Then (6.6) implies that the functions $\ell_{j}(t), j \in \mathcal{J}_{k}\left(t_{0}\right)$, have a local minimum at $t_{0}$, too. Thus, $v_{j}\left(t_{0}\right)^{*} H_{1} v_{j}\left(t_{0}\right)=\ell_{j}^{\prime}\left(t_{0}\right)=0$ for all $j \in \mathcal{J}_{k}\left(t_{0}\right)$. The latter combined with Fact 1 yields that $v_{i}\left(t_{0}\right)^{*} H_{1} v_{j}\left(t_{0}\right)=0$ for all $i, j \in \mathcal{J}_{k}\left(t_{0}\right)$. Hence, $v^{*} H_{1} w=0$ for all $v, w \in \mathcal{E}_{k}\left(H\left(t_{0}\right)\right)=\operatorname{span}\left\{v_{j}\left(t_{0}\right) ; j \in \mathcal{J}_{k}\left(t_{0}\right)\right\}$. This completes the proof of (a).

In order to show (b) we consider the pencil $\tilde{H}(t)=H_{1}+t H_{0}$. Note that $H(t)=t \tilde{H}(1 / t)$ for $t \neq 0$. Let $\tilde{v}_{j}: \mathbb{R} \rightarrow \mathbb{C}^{n}, \tilde{\ell}_{j}: \mathbb{R} \rightarrow \mathbb{R}$ be analytic functions, such that the vectors $\tilde{v}_{j}(t)$ form an orthonormal basis of eigenvectors of $\tilde{H}(t)$ with corresponding eigenvalues $\tilde{\ell}_{j}(t)$. Let $j_{1}, \ldots, j_{p} \in\{1, \ldots, n\}$ denote the indices $j$ for which $\ell_{j}(0)=$ $\lambda_{k}(\tilde{H}(0))=\lambda_{k}\left(H_{1}\right)$. Then the columns of the matrix $V_{1}:=\left[\tilde{v}_{j_{1}}(0), \ldots, \tilde{v}_{j_{p}}(0)\right]$ form an orthonormal basis of $\mathcal{E}_{k}(\tilde{H}(0))=\mathcal{E}_{k}\left(H_{1}\right)$. Furthermore, by Facts 1 and 2 (applied to the pencil $\tilde{H}(t)$ at $t=0)$ the matrix $G_{1}:=\left[\tilde{v}_{j_{\alpha}}(0)^{*} H_{0} \tilde{v}_{j_{\beta}}(0)\right]_{\alpha, \beta=1, \ldots, p}=V_{1}^{*} H_{0} V_{1}$ is a

Copyright (c) by SIAM. Unauthorized reproduction of this article is prohibited. 
diagonal matrix whose diagonal elements are the derivatives $\tilde{\ell}_{j_{1}}^{\prime}(0), \ldots, \tilde{\ell}_{j_{p}}^{\prime}(0)$. Let $V \in$ $\mathbb{C}^{n \times p}$ be another matrix whose columns form an orthonormal basis of $\mathcal{E}_{k}\left(H_{1}\right)$. Then $V=$ $V_{1} U$ for some unitary matrix $U \in \mathbb{C}^{p \times p}$. Hence, the matrix $G:=V^{*} H_{0} V=U^{*} G_{1} U$ is similar to $G_{1}$. Thus, the derivatives $\tilde{\ell}_{j_{1}}^{\prime}(0), \ldots, \tilde{\ell}_{j_{p}}^{\prime}(0)$ are the eigenvalues of $G$. Assume now w.l.o.g. that $\tilde{\ell}_{j_{1}}(t)=\max \left\{\tilde{\ell}_{j_{\alpha}}(t) ; \alpha=1, \ldots, p\right\}$ for $t \in[0, \epsilon]$ and some $\epsilon>0$. Then $\tilde{\ell}_{j_{1}}^{\prime}(0)=\max \left\{\ell_{j_{\alpha}}^{\prime}(0) ; \alpha=1, \ldots, p\right\}=\lambda_{\max }\left(G_{1}\right)=\lambda_{\max }(G)$. Assume further that $k=1$ or $\lambda_{k-1}\left(H_{1}\right)>\lambda_{k}\left(H_{1}\right)$. Then $\tilde{\ell}_{j_{1}}(t)=\lambda_{k}(\tilde{H}(t))$ for $t \in[0, \epsilon]$. If additionally $\lambda_{k}\left(H_{1}\right)=0$, then

$$
\lambda_{k}(\tilde{H}(t))=\tilde{\ell}_{j_{1}}(t)=\tilde{\ell}_{j_{1}}^{\prime}(0) t+o(t)=\lambda_{\max }(G) t+o(t), \quad \lim _{t \rightarrow 0+} \frac{o(t)}{t}=0 .
$$

It follows that

$$
\lim _{t \rightarrow \infty} \lambda_{k}(H(t))=\lim _{t \rightarrow \infty} t \lambda_{k}\left(\tilde{H}\left(\frac{1}{t}\right)\right)=\lim _{t \rightarrow \infty} t\left(\lambda_{\max }(G) \frac{1}{t}+o\left(\frac{1}{t}\right)\right)=\lambda_{\max }(G) .
$$

This concludes the proof of (b).

Some of the assertions of Proposition 6.1 were shown in [9]. The complete proof was given here for the convenience of the reader.

We are now in a position to prove Theorem 3.1. To this end we introduce the notation

$$
\begin{aligned}
& m_{h}\left(H_{0}, H_{1}\right):=\sup \left\{v^{*} H_{0} v ; v \in \mathbb{C}^{n}, v^{*} H_{1} v=0,\|v\|=1\right\}, \\
& \tilde{m}_{h}\left(H_{0}, H_{1}\right):=\inf \left\{v^{*} H_{0} v ; v \in \mathbb{C}^{n}, v^{*} H_{1} v=0,\|v\|=1\right\},
\end{aligned}
$$

where $H_{0}, H_{1} \in \operatorname{Herm}(n)$. Then Theorem 2.1 states that

$$
\mu_{\mathrm{Herm}}(M)=\left(m_{h}\left(M^{*} M, M_{h}\right)\right)^{1 / 2}, \quad \tilde{\mu}_{\mathrm{Herm}}(M)=\left(\tilde{m}_{h}\left(M^{*} M, M_{h}\right)\right)^{1 / 2}
$$

for any $M \in \mathbb{C}^{n \times n}$ for which the matrix $M_{h}=i\left(M-M^{*}\right)$ is not definite. Thus, Theorem 3.1 is obtained by substituting $M^{*} M$ for $H_{0}$ and $M_{h}$ for $H_{1}$ in the following general result.

Theorem 6.2. Let $H_{0}, H_{1} \in \operatorname{Herm}(n)$, and

$$
\begin{aligned}
& \phi(t)=\lambda_{\max }\left(H_{0}+t H_{1}\right), \\
& \tilde{\phi}(t)=\lambda_{\min }\left(H_{0}+t H_{1}\right), \quad t \in \mathbb{R} .
\end{aligned}
$$

Then the function $\phi$ is convex, the function $\tilde{\phi}$ is concave, and

$$
\begin{aligned}
& m_{h}\left(H_{0}, H_{1}\right)=\inf _{t \in \mathbb{R}} \phi(t), \\
& \tilde{m}_{h}\left(H_{0}, H_{1}\right)=\sup _{t \in \mathbb{R}} \tilde{\phi}(t) .
\end{aligned}
$$

Furthermore, the following statements hold.

(i) If $H_{1}$ is indefinite, then the infimum is attained in the interval $\left[t_{1}, t_{2}\right]$ and the supremum is attained in the interval $\left[-t_{2},-t_{1}\right]$, where

Copyright ( by SIAM. Unauthorized reproduction of this article is prohibited. 


$$
t_{1}=\frac{\lambda_{\max }\left(H_{0}\right)-\lambda_{\min }\left(H_{0}\right)}{\lambda_{\min }\left(H_{1}\right)}, \quad t_{2}=\frac{\lambda_{\max }\left(H_{0}\right)-\lambda_{\min }\left(H_{0}\right)}{\lambda_{\max }\left(H_{1}\right)} .
$$

(ii) Suppose $H_{1}$ is positive (negative) semidefinite but not definite. Then the functions $\phi(\cdot)$ and $\tilde{\phi}(\cdot)$ are both increasing (both decreasing). Moreover, we have

$$
\begin{aligned}
m_{h}\left(H_{0}, H_{1}\right) & =\lambda_{\max }\left(V^{*} H_{0} V\right) \\
& =\left\{\begin{array}{cc}
\lim _{t \rightarrow-\infty} \phi(t) & \text { if } H_{1} \text { is positive semidefinite, } \\
\lim _{t \rightarrow \infty} \phi(t) & \text { if } H_{1} \text { is negative semidefinite, }
\end{array}\right. \\
\tilde{m}_{h}\left(H_{0}, H_{1}\right) & =\lambda_{\min }\left(V^{*} H_{0} V\right) \\
& =\left\{\begin{array}{cc}
\lim _{t \rightarrow \infty} \tilde{\phi}(t) & \text { if } H_{1} \text { is positive semidefinite, } \\
\lim _{t \rightarrow-\infty} \tilde{\phi}(t) & \text { if } H_{1} \text { is negative semidefinite, }
\end{array}\right.
\end{aligned}
$$

where $V$ is any matrix whose columns form an orthonormal basis of ker $H_{1}$.

(iii) Suppose $H_{1}$ is positive (negative) definite. Then the functions $\phi(\cdot)$ and $\tilde{\phi}(\cdot)$ are both strictly increasing (both strictly decreasing). Moreover, we have

$$
\begin{aligned}
& m_{h}\left(H_{0}, H_{1}\right)=-\infty, \\
& \tilde{m}_{h}\left(H_{0}, H_{1}\right)=\infty .
\end{aligned}
$$

Proof. It suffices to show the statements about $\phi$ and $m_{h}\left(H_{0}, H_{1}\right)$. The statements about $\tilde{\phi}$ and $\tilde{m}_{h}\left(H_{0}, H_{1}\right)$ then follow immediately using the facts that $\lambda_{\min }(H)=$ $-\lambda_{\max }(-H)$ and $\tilde{m}_{h}\left(H_{0}, H_{1}\right)=-m_{h}\left(-H_{0},-H_{1}\right)$ for all $H, H_{0}, H_{1} \in \operatorname{Herm}(n)$.

The well-known convexity of the function $H \mapsto \lambda_{\max }(H), H \in \operatorname{Herm}(n) \quad[3$, Example 3.10] implies the convexity of $\phi$. Furthermore, by (6.2) the following inequalities hold:

$$
\begin{gathered}
\lambda_{\min }\left(H_{0}\right)+\lambda_{\max }\left(t H_{1}\right) \leq \phi(t) \leq \lambda_{\max }\left(H_{0}\right)+\lambda_{\max }\left(t H_{1}\right), \\
\phi\left(t_{*}\right)+\lambda_{\min }\left(t H_{1}\right) \leq \phi\left(t_{*}+t\right) \leq \phi\left(t_{*}\right)+\lambda_{\max }\left(t H_{1}\right), \quad t, t_{*} \in \mathbb{R} .
\end{gathered}
$$

Note that

$$
\lambda_{\text {max }}\left(t H_{1}\right)= \begin{cases}\lambda_{\text {max }}\left(H_{1}\right) t & \text { if } t \geq 0, \\ \lambda_{\min }\left(H_{1}\right) t & \text { if } t \leq 0 .\end{cases}
$$

The monotonicity statements about $\phi$ in (ii) and (iii) follow from (6.10). Next we show the identity (6.7). For any unit vector $v \in \mathbb{C}^{n}$ satisfying $v^{*} H_{1} v=0$ and any $t \in \mathbb{R}$ we have by the Courant-Fischer theorem that $v^{*} H_{0} v=v^{*}\left(H_{0}+t H_{1}\right) v \leq \phi(t)$. This implies

$$
m_{h}\left(H_{0}, H_{1}\right) \leq \inf _{t \in \mathbb{R}} \phi(t) .
$$

In order to show the opposite inequality we now distinguish four cases.

Case 1. $H_{1}$ is indefinite and $\lambda_{\min }\left(H_{0}\right)<\lambda_{\max }\left(H_{0}\right)$. Let $t_{1}, t_{2}$ be defined as in (6.8). Then $t_{1}<0<t_{2}$, and (6.11) yields that $\phi(0)=\lambda_{\min }\left(H_{0}\right)+\lambda_{\max }\left(t_{j} H_{1}\right), j=1,2$. By combining this with the left inequality in $(6.9)$ we obtain $\phi(0) \leq \phi\left(t_{j}\right)$. Consequently, the continuous function $\phi(\cdot)$ attains a local minimum at some $t_{0}$ in the open interval $\left(t_{1}, t_{2}\right)$. By claim (a) of Proposition 6.1 there exists a unit vector $v_{0}$ satisfying 
$\left(H_{0}+t_{0} H_{1}\right) v_{0}=\phi\left(t_{0}\right) v_{0}$ and $v_{0}^{*} H_{1} v_{0}=0$, whence $v_{0}^{*} H_{0} v_{0}=\phi\left(t_{0}\right)$. Thus, $\inf _{t \in \mathbb{R}} \phi(t) \leq$ $\phi\left(t_{0}\right) \leq m_{h}\left(H_{0}, H_{1}\right)$. Thus, equality holds in (6.12).

Case 2. $H_{1}$ is indefinite and $\lambda_{\min }\left(H_{0}\right)=\lambda_{\max }\left(H_{0}\right)$. In this case $H_{0}$ is a scalar multiple of the identity matrix: $H_{0}=c I$ with $c \in \mathbb{R}$. Hence, $\phi(t)=c+\lambda_{\max }\left(t H_{1}\right)$, and (6.11) yields $\inf _{t \in \mathbb{R}} \phi(t)=\phi(0)=c$. On the other hand we have $v^{*} H_{0} v=c$ for all unit vectors $v$. Moreover, since $H_{1}$ is indefinite there exists a unit vector $v$ satisfying $v^{*} H_{1} v=0$. Thus, $m_{h}\left(H_{0}, H_{1}\right)=c$.

Case 3. $H_{1}$ is semidefinite but not definite. Then $v^{*} H_{1} v=0$ implies $v \in$ ker $H_{1} \neq\{0\}$. Let $V$ be a matrix whose columns form an orthonormal basis of ker $H_{1}$. Then

$$
m_{h}\left(H_{0}, H_{1}\right)=\max \left\{v^{*} H_{0} v ; v \in \operatorname{ker} H_{1},\|v\|=1\right\}=\lambda_{\max }\left(V^{*} H_{0} V\right) .
$$

On the other hand claim (b) of Proposition 6.1 yields

$$
\lambda_{\max }\left(V^{*} H_{0} V\right)= \begin{cases}\lim _{t \rightarrow \infty} \phi(t) & \text { if } H_{1} \text { is negative semidefinite, } \\ \lim _{t \rightarrow-\infty} \phi(t) & \text { if } H_{1} \text { is positive semidefinite. }\end{cases}
$$

It follows that $\inf _{t \in \mathbb{R}} \phi(t) \leq \lambda_{\max }\left(V^{*} H_{0} V\right)=m_{h}\left(H_{0}, H_{1}\right)$. The latter inequality is actually an equality because of (6.12).

Case 4. $H_{1}$ is definite. Then $m_{h}\left(H_{0}, H_{1}\right)=-\infty$ by definition. Moreover, (6.9) yields that

$$
-\infty= \begin{cases}\lim _{t \rightarrow-\infty} \phi(t) & \text { if } H_{1} \text { is positive definite } \\ \lim _{t \rightarrow \infty} \phi(t) & \text { if } H_{1} \text { is negative definite. }\end{cases}
$$

Thus, (6.7) holds in this case.

Next, we prove Theorem 3.3. For $H \in \operatorname{Herm}(n), S \in \operatorname{Sym}(n)$, we define

$$
\begin{aligned}
& m_{h s}(H, S):=\sup \left\{v^{*} H v ; v \in \mathbb{C}^{n}, v^{\top} S v=0,\|v\|=1\right\}, \\
& \tilde{m}_{h s}(H, S):=\inf \left\{v^{*} H v ; v \in \mathbb{C}^{n}, v^{\top} S v=0,\|v\|=1\right\} .
\end{aligned}
$$

Then Theorem 2.1 states that for any $M \in \mathbb{C}^{n \times n}, n \geq 2$,

$$
\begin{aligned}
& \mu_{\text {Skew }}(M)=\left(m_{h s}\left(M^{*} M, M_{s}\right)\right)^{1 / 2}, \\
& \tilde{\mu}_{\text {Skew }}(M)=\left(\tilde{m}_{h s}\left(M^{*} M, M_{s}\right)\right)^{1 / 2},
\end{aligned}
$$

where $M_{s}=M+M^{\top}$. Thus, Theorem 3.3 is obtained by substituting $M^{*} M$ for $H$ and $M_{s}$ for $S$ in the result below.

Theorem 6.3. $H \in \operatorname{Herm}(n), S \in \operatorname{Sym}(n)$. For $t \in \mathbb{R}$, let

$$
F(t)=\left[\begin{array}{ll}
H & t \bar{S} \\
t S & \bar{H}
\end{array}\right] \in \operatorname{Herm}(2 n)
$$

and

$$
\psi(t)=\lambda_{2}(F(t)), \quad \tilde{\psi}(t)=\lambda_{2 n-1}(F(t)) .
$$

Then the functions $\psi(\cdot)$ and $-\tilde{\psi}(\cdot)$ are both unimodal on $[0, \infty)$, and

Copyright ( by SIAM. Unauthorized reproduction of this article is prohibited. 


$$
\begin{aligned}
& m_{h s}(H, S)=\inf _{t \in[0, \infty)} \psi(t), \\
& \tilde{m}_{h s}(H, S)=\sup _{t \in[0, \infty)} \tilde{\psi}(t) .
\end{aligned}
$$

Furthermore, the following statements hold.

(i) If $\operatorname{rank}(S) \geq 2$, then both the infimum and the supremum are attained in the interval $\left[0, t_{1}\right]$, where $t_{1}=2\|H\| / \sigma_{2}(S)$.

(ii) Suppose $\operatorname{rank}(S)=1$. Then the function $\psi:[0, \infty) \rightarrow \mathbb{R}$ is decreasing, the function $\tilde{\psi}:[0, \infty) \rightarrow \mathbb{R}$ is increasing, and

$$
\begin{aligned}
& m_{h s}(H, S)=\lim _{t \rightarrow \infty} \psi(t)=\lambda_{\max }\left(V^{*} H V\right), \\
& \tilde{m}_{h s}(H, S)=\lim _{t \rightarrow \infty} \tilde{\psi}(t)=\lambda_{\min }\left(V^{*} H V\right),
\end{aligned}
$$

where $V$ is any matrix whose columns form an orthonormal basis of ker $S$.

(iii) If $S=0$, then the functions $\psi(\cdot)$ and $\tilde{\psi}(\cdot)$ are both constant, and

$$
\begin{aligned}
& m_{h s}(H, S)=\lambda_{\max }(H)=\psi(0), \\
& \tilde{m}_{h s}(H, S)=\lambda_{\min }(H)=\tilde{\psi}(0) .
\end{aligned}
$$

It is enough to show the statements about $m_{h s}(H, S)$ and $\psi$. The statements about $\tilde{m}_{h s}(H, S)$ and $\tilde{\psi}$ then follow immediately using the facts that $\tilde{m}_{h s}(H, S)=$ $-m_{h s}(-H,-S)$ and $\lambda_{2 n-1}(F)=-\lambda_{2}(-F)$ for all $H \in \operatorname{Herm}(n), S \in \operatorname{Sym}(n), F \in$ $\operatorname{Herm}(2 n)$. We split the proof into several lemmas, which give some additional information. First note that $F(-t)=T F(t) T^{-1}$, where $T=\left[\begin{array}{cc}-I & 0 \\ 0 & I\end{array}\right]$. Thus, $\psi(t)=\psi(-t)$ for all $t \in \mathbb{R}$.

Lemma 6.1. Forany $H \in \operatorname{Herm}(n), S \in \operatorname{Sym}(n)$, wehave $m_{h s}(H, S) \leq \inf _{t \in[0, \infty)} \psi(t)$. Proof. For a unit vector $v \in \mathbb{C}^{n}$, let

$$
\mathcal{U}_{v}:=\left\{\left[\begin{array}{c}
z_{1} v \\
z_{2}^{-} v
\end{array}\right] ; z_{1}, z_{2} \in \mathbb{C}\right\}
$$

Note that $\mathcal{U}_{v}$ is a 2 -dimensional subspace of $\mathbb{C}^{2 n}$, and

$$
\left[\begin{array}{c}
z_{1} v \\
z_{2}^{-} v
\end{array}\right]^{*} F(t)\left[\begin{array}{c}
z_{1} v \\
z_{2}^{-} v
\end{array}\right]=\left(\left|z_{1}\right|^{2}+\left|z_{2}\right|^{2}\right) v^{*} H v+2 t \Re\left(z_{1} z_{2} v^{\top} S v\right), \quad z_{1}, z_{2} \in \mathbb{C} .
$$

Suppose now that $v^{\top} S v=0$. Then by the Courant-Fischer max-min-principle and (6.16)

$$
\psi(t)=\lambda_{2}(F(t)) \geq \min _{x \in \mathcal{U}_{v},\|x\|=1} x^{*} F(t) x=v^{*} H v \quad \text { for all } t \in \mathbb{R}
$$

Hence, $\psi(t) \geq m_{h s}(H, S)$.

Next, we consider the case that $\psi$ attains its minimum at 0 . To this end we need the lemma below, which has already been used in the proof of Theorem 2.1.

Lemma 6.2. Let $\mathcal{V}$ be a subspace of $\mathbb{C}^{n}$ of dimension $\operatorname{dim} \mathcal{V} \geq 2$. Then to any $S \in \operatorname{Sym}(n)$ there is a nonzero $v \in \mathcal{V}$ satisfying $v^{\top} S v=0$.

Proof. For $z_{1}, z_{2} \in \mathbb{C}$, let $v_{z_{1}, z_{2}}=z_{1} v_{1}+z_{2} v_{2}$, where $v_{1}, v_{2} \in \mathcal{V}$ are linearly independent vectors. The function $\left(z_{1}, z_{2}\right) \mapsto v_{z_{1}, z_{2}}^{\top} S v_{z_{1}, z_{2}}$ is a homogeneous quadratic polynomial and has a zero $\left(z_{1}, z_{2}\right) \neq(0,0)$.

Copyright (C) by SIAM. Unauthorized reproduction of this article is prohibited. 
LEMma 6.3. The following statements are equivalent.

(i) $m_{h s}(H, S)=\psi(0)=\lambda_{\max }(H)$.

(ii) Either $\operatorname{dim} \mathcal{E}_{1}(H) \geq 2$, or $\operatorname{dim} \mathcal{E}_{1}(H)=1$ and $v^{\top} S v=0$ for $v \in \mathcal{E}_{1}(H)$.

(iii) The function $\mathbb{R} \ni t \mapsto \psi(t)$ attains its minimum at $t=0$.

Proof. Let $v_{1}, \ldots, v_{d}$ be a basis of the eigenspace $\mathcal{E}_{k}(H)$. Then

$$
\mathcal{E}_{2 k-1}(F(0))=\mathcal{E}_{2 k}(F(0))=\bigoplus_{j=1}^{d} \mathcal{U}_{v_{j}},
$$

where the subspaces $\mathcal{U}_{v_{j}}$ are defined as in (6.15) and $\bigoplus$ denotes the direct sum. Hence, the eigenspaces of $F(0)$ have even dimension, and $\lambda_{\max }(H)=\psi(0)$.

(i) $\Leftrightarrow$ (ii). Let $K=\left\{v \in \mathbb{C}^{n} ;\|v\|=1, v^{\top} S v=0\right\}$. Then $K$ is compact and $m_{h s}(H, S)=\max _{v \in K} v^{*} H v$. Obviously, $m_{h s}(H, S) \leq \max \left\{v^{*} H v ; v \in \mathbb{C}^{n},\|v\|=1\right\}=$ $\lambda_{\max }(H)$. For any unit vector $v$, we have $v^{*} H v=\lambda_{\max }(H)$ iff $v \in \mathcal{E}_{1}$. Thus, if $K \cap \mathcal{E}_{1}(H)=\varnothing$, then $v^{*} H v<\lambda_{\max }(H)$ for all $v \in K$, whence $m_{h s}(H, S)<\lambda_{\max }(H)$. On the other hand if $K \cap \mathcal{E}_{1}(H) \neq \varnothing$, then $v^{\top} S v=0$ and $v^{*} H v=\lambda_{\max }(H)$ for some unit vector $v$, whence $m_{h s}(H, S)=\lambda_{\max }(H)$. By Lemma 6.2 we have $K \cap \mathcal{E}_{1}(H) \neq \varnothing$ if $\operatorname{dim} \mathcal{E}_{1}(H) \geq 2$. The implication (i) $\Rightarrow$ (iii) follows from Lemma 6.1. (iii) $\Rightarrow$ (i). Since (i) is satisfied if $\operatorname{dim} \mathcal{E}_{1}(H) \geq 2$, we may assume that $\operatorname{dim} \mathcal{E}_{1}(H)=1$. Then $\mathcal{E}_{2}(F(0))=$ $\mathcal{E}_{1}(F(0))=\mathcal{U}_{v}$ for a unit vector $v \in \mathcal{E}_{1}(H)$, and $\lambda_{2}(F(0))>\lambda_{3}(F(0))$. Hence, (iii) and claim (a) of Proposition 6.1 yield that $x^{*}\left[\begin{array}{ll}0 & \bar{S} \\ 0\end{array}\right] x=0$ for all $x \in \mathcal{E}_{2}(F(0))$. In other words we have for all $z_{1}, z_{2} \in \mathbb{C}$,

$$
0=\left[\begin{array}{l}
z_{1} v \\
z_{2} v
\end{array}\right]^{*}\left[\begin{array}{cc}
0 & \bar{S} \\
S & 0
\end{array}\right]\left[\frac{z_{1} v}{z_{2} v}\right]=2 \Re\left(z_{1} z_{2} v^{\top} S v\right) .
$$

This implies $v^{\top} S v=0$. Thus, $m_{h s}(H, S)=v^{*} H v=\psi(0)$.

Lemma 6.4. Suppose the function $\mathbb{R} \ni t \mapsto \psi(t)$ attains a local extremum at $t_{0} \neq 0$. Then there is a unit vector $v \in \mathbb{C}^{n}$ satisfying $v^{*} H v=\psi\left(t_{0}\right)$ and $v^{\top} S v=0$.

Proof. If the assumption of the lemma holds, then by Proposition 6.1 there is a nonzero $v_{0} \in \mathbb{C}^{2 n}$ such that

$$
\begin{gathered}
F\left(t_{0}\right) v_{0}=\psi\left(t_{0}\right) v_{0}, \\
v_{0}^{*}\left[\begin{array}{cc}
0 & \bar{S} \\
S & 0
\end{array}\right] v_{0}=0 .
\end{gathered}
$$

Let

$$
H_{0}:=H-\psi\left(t_{0}\right) I, \quad v_{0}=\left[\begin{array}{l}
x \\
\bar{y}
\end{array}\right], \quad x, y \in \mathbb{C}^{n} .
$$

Then (6.17) is equivalent to the equations

$$
H_{0} x=-t_{0} \overline{S y}, \quad \overline{H_{0} y}=-t_{0} S x
$$

which imply

Copyright $@$ by SIAM. Unauthorized reproduction of this article is prohibited. 


$$
\begin{aligned}
& x^{*} H_{0} x=-t_{0} \overline{x^{\top} S y}=y^{*} H_{0} y, \\
& x^{*} H_{0} y=-t_{0} \overline{x^{\top} S x}=-t_{0} y^{\top} S y .
\end{aligned}
$$

Since $t_{0} \neq 0$ it follows that

$$
\begin{gathered}
x^{\top} S y \in \mathbb{R}, \\
y^{\top} S y=\overline{x^{\top} S x} .
\end{gathered}
$$

Relation (6.18) states that $2 \mathfrak{R}\left(x^{\top} S y\right)=0$. Thus, (6.21) yields

$$
x^{\top} S y=0 .
$$

Now let

$$
\beta:= \begin{cases}1 & \text { if } x^{T} S x=0 \\ i \frac{x^{T} S x}{\left|x^{T} S x\right|} & \text { otherwise }\end{cases}
$$

Then we have

$$
\begin{aligned}
(x \pm \beta y)^{\top} S(x \pm \beta y) & =x^{T} S x+\beta^{2} y^{\top} S y \pm 2 \beta x^{\top} S y \\
& =x^{\top} S x+\underbrace{\beta^{2} \overline{x^{\top} S x}}_{=-x^{T} S x} \pm 2 \beta \underbrace{x^{\top} S y}_{=0} \quad \text { (using (6.22) and (6.23)) } \\
& =0,
\end{aligned}
$$

and

$$
\begin{aligned}
(x \pm \beta y)^{*} H_{0}(x \pm \beta y)= & x^{*} H_{0} x+|\beta|^{2} y^{*} H_{0} y \pm 2 \Re\left(x^{*} H_{0} y \beta\right) \\
= & -t_{0}(\left(1+|\beta|^{2}\right) \overline{x_{=0}^{\top} S y} \pm 2 \underbrace{\mathfrak{R}\left(\overline{x^{\top} S x} \beta\right)}_{=0}) \\
& \quad(\operatorname{using}(6.20) \text { and }(6.23)) \\
= & 0 .
\end{aligned}
$$

At least one of the vectors $x \pm \beta y$ is nonzero and can therefore be divided by its norm. The resulting vector $v \in \mathbb{C}^{n}$ has the required properties.

Corollary 6.4. Suppose the function $\mathbb{R} \ni t \mapsto \psi(t)$ attains a local extremum at $t_{0}>0$. Then $\psi\left(t_{0}\right)=m_{h s}(H, S)=\inf _{t \in[0, \infty)} \psi(t)$.

Proof. We have $m_{h s}(H, S) \leq \inf _{t \in[0, \infty)} \psi(t) \leq \psi\left(t_{0}\right) \leq m_{h s}(H, S)$. The first of these inequalities is Lemma 6.1. The third is a consequence of Lemma 6.4.

Corollary 6.4 in particular states that the function $\psi(\cdot)$ is unimodal on $[0, \infty)$.

Now we treat the three cases $\operatorname{rank}(S) \geq 2, \operatorname{rank}(S)=1$, and $S=0$ separately.

Case 1. $\operatorname{rank}(S) \geq 2$. Let $t_{1}=2\|H\| / \sigma_{2}(S)$. The eigenvalues of $\left[\begin{array}{cc}0 & t \bar{S} \\ t S & 0\end{array}\right]=$ $\left[\begin{array}{cc}0 & t S^{*} \\ t S & 0\end{array}\right]$ are the singular values of $S$ and their negatives. In particular

$$
\lambda_{2}\left(\left[\begin{array}{cc}
0 & t \bar{S} \\
t S & 0
\end{array}\right]\right)=\sigma_{2}(t S)=|t| \sigma_{2}(S)
$$

Copyright @ by SIAM. Unauthorized reproduction of this article is prohibited. 
We conclude that

$$
\psi(t)=\lambda_{2}\left(\left[\begin{array}{lll}
H & t \bar{S} & \\
t S & & \bar{H}
\end{array}\right]\right) \geq \lambda_{2}\left(\left[\begin{array}{cc}
0 & t \bar{S} \\
t S & 0
\end{array}\right]\right)+\lambda_{\min }\left(\left[\begin{array}{cc}
H & 0 \\
0 & \bar{H}
\end{array}\right]\right) \geq|t| \sigma_{2}(S)-\|H\| .
$$

Thus, if $|t|>t_{1}$, then $\psi(t)>\|H\| \geq \lambda_{\max }(H)=\psi(0)$. Consequently, $\psi$ attains its minimum at some $t_{0} \in \mathbb{R}$ with $\left|t_{0}\right| \leq t_{1}$. Since $\psi(t)=\psi(-t)$ there exists a minimizer $t_{0} \geq 0$. If $t_{0}=0$, then $\psi\left(t_{0}\right)=m_{h s}(H, S)=\inf _{t \in[0, \infty)} \psi(t)$ by Lemma 6.3 . If $t_{0}>0$, then the latter chain of equalities holds by Corollary 6.4 .

Case 2. $\operatorname{rank}(S)=1$. In this case $S$ can be written in the form $S=x x^{\top}$ for some nonzero $x \in \mathbb{C}^{n}$. Let $V$ be a matrix whose columns form an orthonormal basis of ker $S=\left\{v \in \mathbb{C}^{n} ; x^{\top} v=0\right\}$. Since $v^{\top} S v=\left(x^{\top} v\right)^{2}$ we have $v^{\top} S v=0$ ff $v \in \operatorname{ker} S=$ range $(V)$. This yields

$$
m_{h s}(H, S)=\max \left\{v^{*} H v ; v \in \operatorname{range}(V),\|v\|=1\right\}=\lambda_{\max }\left(V^{*} H V\right) .
$$

The columns of $\left[\begin{array}{cc}0 & V \\ \bar{V} & 0\end{array}\right]$ form an orthonormal basis of $\operatorname{ker}\left(\left[\begin{array}{cc}0 & \bar{S} \\ S & 0\end{array}\right]\right)$. The nonzero eigenvalues of $\left[\begin{array}{cc}0 & \bar{S} \\ S & 0\end{array}\right]$ are $\pm|x|^{2}$. Thus, $\lambda_{2}\left(\left[\begin{array}{cc}0 & \bar{S} \\ S & 0\end{array}\right]\right)=0$ since we assume $n \geq 2$. Therefore, by claim (b) of Proposition 6.1

$$
\lim _{t \rightarrow \infty} \psi(t)=\lambda_{\max }\left(\left[\begin{array}{cc}
0 & V \\
\bar{V} & 0
\end{array}\right]^{*}\left[\begin{array}{cc}
H & 0 \\
0 & \bar{H}
\end{array}\right]\left[\begin{array}{cc}
0 & V \\
0 & \bar{V}
\end{array}\right]\right)=\lambda_{\max }\left(V^{*} H V\right)
$$

By combining $(6.24),(6.25)$, and Lemma 6.1 we find that $m_{h s}(H, S)=\inf _{t \in[0, \infty)} \psi(t)=$ $\lim _{t \rightarrow \infty} \psi(t)=\lambda_{\max }\left(V^{*} H V\right)$. It remains to show that $\psi$ is decreasing. However, this is immediate from the inequality $\psi(0) \geq m_{h s}(H, S)=\lim _{t \rightarrow \infty} \psi(t)$ and Corollary 6.4.

Case 3. $S=0$. In this case the function $\psi$ is constant and the identities $m_{h s}(H, S)=$ $\lambda_{\max }(H)=\psi(0)=\inf _{t \in[0, \infty)} \psi(t)$ are obvious.

This concludes the proofs of Theorems 6.3 and 3.3.

Acknowledgment. The author thanks the referees and Daniel Kressner for valuable comments on an earlier version of this paper.

\section{REFERENCES}

[1] G. J. Balas, J. C. Doyle, K. Glover, A. Packard, and R. Smith, $\mu$-Analysis and Synthesis Toolbox. User's Guide, The MathWorks Inc., South Natick, MA, 1991.

[2] B. Bernhardsson, A. Rantzer, and L. Qiu, Real perturbation values and real quadratic forms in a complex vector space, Linear Algebra Appl., 270 (1998), pp. 131-154.

[3] S. Boyd and L. Vandenberghe, Convex Optimization, Cambridge University Press, Cambridge, UK, 2004.

[4] M. J. Chen, K. N. Fan, And C. N. Nett, Structured singular values with nondiagonal structures-Part I: Characterizations, Part II: Computation, IEEE Trans. Automat. Control, 41 (1996), pp. 1507-1516.

[5] J. C. Doyle, Analysis of feedback systems with structured uncertainties, IEE Proc., Part D, 129 (1982), pp. $242-250$.

[6] E. Gallestey, Computing spectral value sets using the subharmonicity of the norm of rational matrices, BIT, 38 (1998), pp. 22-33.

[7] D. Hinrichsen and A. J. Pritchard, Real and complex stability radii: A survey, in Control of Uncertain Systems, D. Hinrichsen and B. Mårtensson, eds., Birkhäuser, Basel, 1990, pp. 119-162.

[8] D. Hinrichsen and A. J. Pritchard, Mathematical Systems Theory I. Modelling, State Space Analysis, Stability and Robustness, Springer-Verlag, Berlin, 2005.

Copyright (c) by SIAM. Unauthorized reproduction of this article is prohibited. 
[9] T. Hu AND L. QIU, On structured perturbation of Hermitian matrices, Linear Algebra Appl., 275-276 (1998), pp. 287-314.

[10] M. Karow, Geometry of Spectral Value Sets, Ph.D. thesis, University of Bremen, Bremen, Germany, 2003.

[11] M. Karow, Structured pseudospectra for small perturbations, SIAM J. Matrix Anal. Appl., to appear.

[12] M. Karow, D. Hinrichsen, and A. J. Pritchard, Interconnected systems with uncertain couplings: explicit formulae for $\mu$-values, spectral value sets and stability radii, SIAM J. Control Optim., 45 (2006), pp. 856-884.

[13] M. Karow, E. Kokiopoulou, and D. Kressner, On the computation of structured singular values and pseudospectra, Systems Control Lett., 59 (2010), pp. 122-129.

[14] M. Karow and D. Kressner, On the structured distance to uncontrollability, Systems Control Lett., 58 (2009), pp. 128-132.

[15] M. Karow, D. Kressner, and F. Tisseur, Structured eigenvalue condition numbers, SIAM J. Matrix Anal. Appl., 28 (2006), pp. 1052-1068.

[16] T. Kaто, Perturbation Theory for Linear Operators, 2nd ed., Springer, Berlin, Heidelberg, New York, 1976.

[17] R. A. Lippert, Fixing two eigenvalues by a minimal perturbation, Linear Algebra Appl., 406 (2005), pp. $177-200$

[18] N. Mackey, D. S. Mackey, and F. Tisseur, Structured mapping problems for matrices associated with scalar products part I: Lie and Jordan algebras, SIAM J. Matrix Anal. Appl., 29 (2008), pp. 1389-1410.

[19] A. N. Malyshev, A formula for the 2-norm distance from a matrix to the set of matrices with multiple eigenvalues, Numer. Math., 83 (1999), pp. 443-454.

[20] A. Packard and J. C. Doyle, The complex structured singular value, Automatica J. IFAC, 29 (1993), pp. 71-109.

[21] E. Polak, Computational Methods in Optimization: A Unified Approach, Academic Press, New York, 1971.

[22] L. Qiu, B. Bernhardsson, A. Rantzer, E. J. Davison, P. M. Young, and J. C. Doyle, A formula for computation of the real stability radius, Automatica J. IFAC, 31 (1995), pp. 879-890.

[23] M. G. Safonov and K.-H. M. Fan, Special issue: Multivariable stability margin, Int. J. Robust Nonlinear Control, 7 (1997), pp. 97-226.

[24] L. N. Trefethen and M. Embree, Spectra and Pseudospectra: The Behavior of Nonnormal Matrices and Operators, Princeton University Press, Princeton, NJ, 2005.

[25] K. Zhou, J. C. Doyle, and K. Glover, Robust and Optimal Control, Prentice Hall, Upper Saddle River, NJ, 1996.

Copyright (C) by SIAM. Unauthorized reproduction of this article is prohibited. 